\title{
Optical surface photometry of radio galaxies
}

\author{
II. Observations and data analysis \\ F. Govoni ${ }^{1,3}$, R. Falomo ${ }^{1}$, G. Fasano ${ }^{1}$, and R. Scarpa ${ }^{2}$ \\ 1 Osservatorio Astronomico di Padova, vicolo dell'Osservatorio 5, I-35122 Padova, Italy \\ 2 Space Telescope Science Institute, 3700 San Martin Drive, Baltimore, MD 21218, U.S.A. \\ 3 Dip. di Astronomia, Univ. di Bologna, Via Ranzani 1, I-40127 Bologna, Italy
}

Received July 23, 1999; accepted January 24, 2000

\begin{abstract}
Optical imaging observations for 50 radio galaxies are presented. For each object isophotal contours, photometric profiles, structural parameters (position angle, ellipticity, Fourier coefficients), and total magnitudes are given. These observations, obtained in the Cousins $R$ band, complement the data presented in a previous paper and are part of a larger project aimed at studying the optical properties of low redshift $(z \leq 0.12)$ radio galaxies (Govoni et al. 1999). Comments for each individual source are reported.
\end{abstract}

Key words: galaxies: active — galaxies: fundamental parameters - galaxies: photometry

\section{Introduction}

Only a small fraction of elliptical galaxies emit at radio wavelengths. This is probably due to the combination of two factors: 1) the lifetime of the radio source is much less than the typical lifetime of the host galaxy; 2) not all elliptical galaxies may harbor the conditions for radio activity. Therefore, a detailed analysis and comparison of the properties of radio and non-radio galaxies are of great relevance for understanding the phenomenon. Previous optical studies (Hine \& Longair 1979; Longair \& Seldner 1979; Lilly \& Prestage 1987; Prestage \& Peacock 1988; Owen \& Laing 1989; Smith \& Heckman 1989a,b; Owen \& White 1991; Gonzalez-Serrano et al. 1993; de Juan et al. 1994; Colina \& de Juan 1995; Ledlow \& Owen 1995) have investigated the role of galaxy-galaxy interaction in the creation and

Send offprint requests to: F. Govoni,

e-mail: fgovoni@astbo1.bo.cnr.it

Based on observations collected at the European Southern

Observatory, La Silla, Chile, and at the Nordic Optical Telescope, La Palma. fueling of nuclear radio sources, as well as the connection between overall galaxy properties (e.g. luminosity and scale length) and radio morphology.

In order to contribute to the study of the photometrical and morphological properties of the galaxies hosting radio sources, we have undertaken a systematic study of low redshift radio galaxies in the optical band.

In a previous paper (Fasano et al. 1996; hereafter Paper I) we presented structural (position angle, ellipticity, Fourier coefficients) and photometric profiles together with isophotal contours in the $B$ and $R$ bands for 29 galaxies extracted from a complete sample of 95 radio galaxies in the redshift range $0.01 \leq z \leq 0.12$.

Here, we give the results for 50 more galaxies observed in the Cousins $R$ band, bringing to $79,83 \%$ of the original sample, the total number of sources for which we were able to secure data of the required quality. A full discussion of the astrophysical implications of these observations is given in Govoni et al. (1999), where the overall properties of galaxies hosting radio emission are compared with radio quiet ellipticals.

\section{Observations and data analysis}

\subsection{The sample}

The sample is composed of radio galaxies in the redshift range $0.01 \leq z \leq 0.12$ extracted from two complete surveys of radio sources.

The first one is the all sky survey of radio sources with radio flux at $2.7 \mathrm{GHz}$ greater than 2 Jy by Wall \& Peacock (1985; hereafter WP). From this survey we extracted all objects, in the above redshift range, classified as radio galaxies (see also Tadhunter et al. 1993) at declination $\delta<10^{\circ}$.

The second list is the Ekers et al. (1989; hereafter EK) catalogue of radio galaxies with flux at $2.7 \mathrm{GHz}$ greater 
Table 1. The sample

\begin{tabular}{|c|c|c|c|c|c|c|c|c|c|}
\hline $\begin{array}{l}\text { IAU name } \\
(1)\end{array}$ & $\begin{array}{c}\text { Other name } \\
\text { (2) }\end{array}$ & $\begin{array}{l}\text { Smp. } \\
(3)\end{array}$ & $\begin{array}{c}\mathrm{RA}(2000) \\
(4)\end{array}$ & $\begin{array}{c}\mathrm{DEC}(2000) \\
(5)\end{array}$ & $\begin{array}{c}z \\
(6)\end{array}$ & $\begin{array}{c}K_{R} \\
(7)\end{array}$ & $\begin{array}{l}A_{V} \\
(8)\end{array}$ & $\begin{array}{l}\text { FR } \\
(9)\end{array}$ & $\begin{array}{l}\text { Ref. }^{a} \\
(10)\end{array}$ \\
\hline $0255+058$ & $3 \mathrm{C} 75$ & WP & 025741.5 & +060137 & 0.023 & 0.02 & 0.54 & $\mathrm{I}$ & 1 \\
\hline $0257-398$ & & $\mathrm{EK}$ & 025926.6 & -394037 & 0.066 & 0.06 & 0.10 & II & 2 \\
\hline $0307-305$ & & EK & 031000.9 & -301933 & 0.066 & 0.06 & 0.10 & II & 2 \\
\hline $0312-343$ & & EK & $03 \quad 1432.7$ & -340740 & 0.067 & 0.06 & 0.13 & I & 2 \\
\hline $0325+023$ & $3 \mathrm{C} 88$ & WP & 032753.9 & +023342 & 0.030 & 0.03 & 0.51 & II & 1 \\
\hline $0332-391$ & & EK & 033407.2 & -390004 & 0.063 & 0.05 & 0.10 & I & 2,3 \\
\hline $0344-345$ & & EK & 034630.5 & -342247 & 0.053 & 0.04 & 0.06 & I & 2,3 \\
\hline $0427-539$ & & WP & 042907.3 & -534940 & 0.038 & 0.03 & 0.17 & I & 1 \\
\hline $0430+052$ & $3 \mathrm{C} 120$ & WP & 043310.5 & +052215 & 0.033 & 0.03 & 0.67 & I & 1 \\
\hline $0434-225$ & OF-257 & EK & 043635.9 & -222634 & 0.069 & 0.06 & 0.19 & I & 2 \\
\hline $0446-206$ & OF- 277.2 & $\mathrm{EK}$ & 044829.9 & -203213 & 0.073 & 0.06 & 0.19 & I & 2 \\
\hline $0452-190$ & & EK & 045417.7 & -185529 & 0.039 & 0.03 & 0.26 & I & 2 \\
\hline $0453-206$ & NGC 1692 & WP & 045523.6 & -203415 & 0.035 & 0.03 & 0.19 & I/II & 1 \\
\hline $0511-305$ & & EK & 051331.2 & -302849 & 0.058 & 0.05 & 0.10 & II & 2,3 \\
\hline $0533-377$ & & EK & 053522.3 & -374313 & 0.096 & 0.09 & 0.16 & I & 2 \\
\hline $0546-329$ & & EK & $05 \quad 4827.4$ & -325837 & 0.037 & 0.03 & 0.16 & I & 2,3 \\
\hline $0548-317$ & & EK & 055049.4 & -314426 & 0.034 & 0.03 & 0.13 & II & 2 \\
\hline $0620-526$ & & WP & 062142.4 & -524132 & 0.051 & 0.04 & 0.30 & $\mathrm{I}$ & 1 \\
\hline $0625-354$ & OH-342 & WP & 062706.4 & -352916 & 0.055 & 0.04 & 0.38 & $\mathrm{I}$ & 2 \\
\hline $0625-536$ & & WP & 062620.4 & -534135 & 0.054 & 0.05 & 0.34 & II & 1 \\
\hline $0634-205$ & & EK & 063632.9 & -203453 & 0.056 & 0.05 & 1.22 & $\mathrm{I} / \mathrm{II}$ & 2 \\
\hline $0712-349$ & & EK & 071356.3 & -350126 & 0.044 & 0.03 & 1.12 & I & 2 \\
\hline $0718-340$ & & EK & 072047.2 & -340705 & 0.029 & 0.02 & 1.28 & $\mathrm{I} / \mathrm{II}$ & 2 \\
\hline $0806-103$ & 3C 195 & WP & 080853.3 & -102740 & 0.110 & 0.10 & 0.58 & II & 1 \\
\hline $0915-118$ & Hyd A, 3C 218 & WP & 091805.5 & -120543 & 0.054 & 0.04 & 0.29 & $\mathrm{I}$ & 1 \\
\hline $0940-304$ & & EK & 094223.2 & -304411 & 0.038 & 0.03 & 0.58 & $\mathrm{I}$ & 2 \\
\hline $0945+076$ & $3 \mathrm{C} 227$ & WP & 094745.6 & +072520 & 0.086 & 0.08 & 0.19 & II & 1 \\
\hline $1002-320$ & & EK & 100439.3 & -321643 & 0.089 & 0.08 & 0.51 & I & 2,3 \\
\hline $1043-290$ & & EK & 104609.7 & -292110 & 0.060 & 0.05 & 0.32 & I & 2 \\
\hline $1053-282$ & OL-288 & EK & 105532.5 & -283133 & 0.061 & 0.05 & 0.32 & I & 2 \\
\hline $1056-360$ & & EK & 105854.6 & -361920 & 0.070 & 0.06 & 0.32 & $\mathrm{I} / \mathrm{II}$ & 2,3 \\
\hline $1107-372$ & NGC 3557 & EK & 110957.4 & -373217 & 0.010 & 0.01 & 0.51 & I & 2 \\
\hline $1123-351$ & & $\mathrm{EK}$ & 112552.5 & -352340 & 0.032 & 0.03 & 0.42 & I & 2 \\
\hline $1251-122$ & $3 \mathrm{C} 278$ & WP & 125435.1 & -123407 & 0.015 & 0.02 & 0.22 & I & 1 \\
\hline $1251-289$ & & EK & 125440.3 & -291339 & 0.057 & 0.05 & 0.38 & I & 2 \\
\hline $1257-253$ & & EK & 125948.8 & -253939 & 0.065 & 0.06 & 0.42 & I & 2 \\
\hline $1258-321$ & & EK & 130100.6 & -322628 & 0.017 & 0.02 & 0.38 & I & 2 \\
\hline $1318-434$ & NGC 5090 & $\mathrm{WP}$ & 132112.4 & -434215 & 0.011 & 0.01 & 0.53 & I & 1 \\
\hline $1323-271$ & & EK & 132610.2 & -272536 & 0.044 & 0.03 & 0.32 & $\mathrm{U}$ & 2 \\
\hline $1333-337$ & IC4296 & WP & 133638.8 & -335756 & 0.013 & 0.01 & 0.26 & $\mathrm{I} / \mathrm{II}$ & $1,2,3$ \\
\hline $1344-241$ & & EK & 134712.1 & -242221 & 0.020 & 0.02 & 0.35 & I & 2 \\
\hline $1354-251$ & & EK & 135716.0 & -252323 & 0.038 & 0.03 & 0.35 & I & 2 \\
\hline $1400-337$ & NGC 5419 & EK & 140338.5 & -335843 & 0.014 & 0.01 & 0.35 & I & 2 \\
\hline $1404-267$ & IC4374 & EK & 140729.4 & -270102 & 0.022 & 0.02 & 0.32 & $\mathrm{I} ?$ & 2 \\
\hline $1637-771$ & PGC 058874 & WP & 164415.7 & -771548 & 0.041 & 0.03 & 0.47 & II & 1,3 \\
\hline $1717-009$ & 3C 353 & WP & 172027.8 & -005846 & 0.031 & 0.03 & 0.61 & II & 1 \\
\hline $1733-565$ & & WP & 173735.3 & -563403 & 0.098 & 0.09 & 0.47 & II & 1 \\
\hline $1928-340$ & PGC 063333 & EK & 193137.3 & -335440 & 0.098 & 0.09 & 0.45 & I & 2 \\
\hline $1949+023$ & 3C 403 & WP & 195216.2 & +023023 & 0.059 & 0.05 & 0.83 & II & 1 \\
\hline $2221-023$ & $3 \mathrm{C} 445$ & WP & 222349.8 & -020613 & 0.057 & 0.05 & 0.32 & II & 1 \\
\hline
\end{tabular}

a Radio classification derived from:

(1) Morganti et al. (1993) or Morganti et al. (1997);

(2) Ekers et al. (1989);

(3) Jones \& McAdam (1992). 
Table 2. Observation run of the sample of radio galaxies

\begin{tabular}{cllc}
\hline Run & \multicolumn{1}{c}{ Date } & \multicolumn{1}{c}{ Instrumentation } & $\begin{array}{c}\text { CCD scale } \\
\text { (" } / \text { pixel })\end{array}$ \\
\hline & & & \\
1 & 1993 July 19-21 & ESO 2.2 m + EFOSC2 & 0.332 \\
2 & 1994 September 10-11 & ESO 2.2 m + EFOSC2 & 0.332 \\
3 & 1996 February 9-11 & ESO 1.5 m Danish+DFOSC & 0.403 \\
4 & 1998 March 30-April 2 & ESO 1.5 m Danish+DFOSC & 0.403 \\
5 & 1998 September 21-24 & NOT-HIRAC & 0.110 \\
\hline
\end{tabular}

than $0.25 \mathrm{Jy}$ and $m_{b}<17.0$, in the declination zone $-40^{\circ}<\delta<-17^{\circ}$. All objects classified as E or S0 in the above redshift range were included in our list. Basic data for the 50 objects presented in this paper are summarized in Table 1. Columns 1 and 2 give for each object IAU and other names, Col. 3 gives the subsample, Cols. 4 and 5 the equatorial coordinates (equinox 2000.0), and Col. 6 the redshift. Columns 7 and 8 report the Kcorrection in the $R$ band and the galactic extinction in the $V$ band. We derived the galactic extinction interpolating the data for galactic hydrogen column density given by Stark et al. (1992) and assuming $A_{V} / E_{B-V}=R=$ 3.2 and $E_{B-V}=N_{\mathrm{H}} / 5.110^{21}$ (Knapp \& Kerr 1974). In Cols. 9 and 10 we give the radio classification and reference.

Based on the radio morphology, sources were divided into FRI and FRII radio classes following the Fanaroff \& Riley scheme (Fanaroff \& Riley 1974). Most sources in the WP sample were imaged by Morganti et al. (1993) with the Very Large Array (VLA) and the Australia Telescope Compact Array (ATCA), while VLA radio images are available for most objects in the EK sample. Objects with transitional properties or with unclear classification are marked as I/II, while a U marks the only unresolved source 1323 - 271. For IC 4374 (labeled with a question mark) we were not able to find a radio image, thus we include it in the FRI class because its radio luminosity at $178 \mathrm{MHz}$ is below $210^{25}$ Watt $/ \mathrm{Hz}$, the dividing luminosity between the two classes.

\subsection{Observations}

Observations were secured in five different observing runs. Beside three galaxies (0307 - 305, 0332 - 391 and $1928-$ $340)$ included here, the results of the imaging in $B$ and $R$ bands obtained with the ESO-2.2 $\mathrm{m}$ telescope during runs 1 and 2 (Table 2) have been reported in Paper I. Data presented here were obtained in three more observing runs (run 3, 4, 5) with either the ESO-Danish $1.5 \mathrm{~m}$ telescope or the Nordic Optical Telescope (NOT). The journal of observations is given in Table 3 , where for each object we report the run of observation, the total integration time, the atmospheric seeing expressed by the full width half maximum (FWHM) of stellar images, and the sky surface brightness, together with its estimated $1 \sigma$ uncertainty. The galaxy total apparent $R$ band magnitude (corrected for galactic extinction) computed by extrapolating to infinity the surface brightness profile is also given. This value does not include the K-correction.

For most objects a short $(\sim 2 \mathrm{~min})$ and a long exposure (Table 3) were obtained, so we also have an unsaturated image of the nuclear region. In a few cases, the presence of bright stars in the field forced us to take several short exposures, subsequently combined to form a final, deep image. Photometric conditions were generally good during the observations, as confirmed by repeated observations of photometric standard stars selected from the Landolt (1992) list. Comparison of the photometric zero point for different nights indicates an average internal photometric accuracy of $5-10 \%$. This, combined with the small uncertainty on the sky surface brightness $(1-2 \%)$, gives a global internal photometric accuracy of the order of $10 \%$. The atmospheric seeing was generally around 1 arcsec, and the CCD pixel size (Table 2) were always sufficiently small to ensure proper sampling of the telescope point spread function (PSF).

\subsection{Data reduction and surface photometry}

Data reduction is extensively described in Paper I. Here we simply remind the reader that the IRAF-ccdred package was used for the basic reduction (bias subtraction, image trimming, flat fielding, cosmic rays, etc.). The dark current turned out to be insignificant and was neglected. After flat fielding, images were characterized by a quite regular sky background, well fitted by a first order polynomial.

Final images are shown in Fig. 1, where it is seen that selected sources cover an area of hundreds of arcseconds square, ideal for two-dimensional isophotal analysis, and can be traced down to a surface brightness of $\mu_{R} \sim 25 \mathrm{mag} / \operatorname{arcsec}^{2}$. It is also evident that these radio galaxies are often observed in highly crowded regions, with stars and/or nearby galaxies projected on-top. Sky subtraction and isophotal analysis (drawing, cleaning and 
Table 3. Journal of observations

\begin{tabular}{|c|c|c|c|c|c|c|}
\hline IAU name & run & $\begin{array}{l}\exp \\
(\mathrm{sec})\end{array}$ & $\begin{array}{l}\text { FWHM } \\
(\operatorname{arcsec})\end{array}$ & $\begin{array}{c}\mu_{\text {sky }} \\
\left(\mathrm{mag} / \operatorname{arcsec}^{2}\right)\end{array}$ & $\begin{array}{c}\sigma_{\text {sky }} \\
\left(\mathrm{mag} / \operatorname{arcsec}^{2}\right)\end{array}$ & $\begin{array}{l}m_{\text {tot }} \\
(\mathrm{mag})\end{array}$ \\
\hline $0255+058$ & 5 & 900 & 1.02 & 20.7 & 0.019 & 12.75 \\
\hline $0257-398$ & 3 & 1200 & 1.33 & 21.2 & 0.005 & 14.38 \\
\hline $0307-305$ & 2 & 900 & 1.40 & 20.5 & 0.007 & 14.70 \\
\hline $0312-343$ & 3 & 1200 & 1.29 & 21.3 & 0.006 & 14.01 \\
\hline $0325+023$ & 5 & 900 & 1.21 & 20.8 & 0.012 & 12.70 \\
\hline $0332-391$ & 2 & 1200 & 1.70 & 20.7 & 0.007 & 14.06 \\
\hline $0344-345$ & 3 & 1500 & 1.44 & 21.1 & 0.004 & 14.70 \\
\hline $0427-539$ & 3 & 600 & 1.13 & 21.2 & 0.005 & 12.69 \\
\hline $0430+052$ & 3 & 1200 & 1.33 & 20.8 & 0.004 & 13.01 \\
\hline $0434-225$ & 3 & 1200 & 1.33 & 21.3 & 0.007 & 13.33 \\
\hline $0446-206$ & 3 & 1200 & 1.16 & 21.1 & 0.005 & 14.81 \\
\hline $0452-190$ & 3 & 1200 & 1.35 & 21.4 & 0.009 & 13.00 \\
\hline $0453-206$ & 3 & 600 & 1.37 & 21.4 & 0.007 & 12.57 \\
\hline $0511-305$ & 3 & 1800 & 1.39 & 21.2 & 0.004 & 14.66 \\
\hline $0533-377$ & 3 & 1800 & 1.49 & 21.4 & 0.009 & 14.57 \\
\hline $0546-329$ & 3 & 1200 & 1.27 & 21.3 & 0.006 & 12.34 \\
\hline $0548-317$ & 3 & 900 & 1.50 & 20.8 & 0.010 & 13.42 \\
\hline $0620-526$ & 3 & 600 & 1.64 & 20.8 & 0.017 & 12.53 \\
\hline $0625-354$ & 3 & 900 & 1.25 & 20.0 & 0.009 & 13.11 \\
\hline $0625-536$ & 3 & 1200 & 1.21 & 20.9 & 0.006 & 12.39 \\
\hline $0634-205$ & 3 & 1800 & 1.31 & 20.9 & 0.008 & 13.90 \\
\hline $0712-349$ & 3 & 1200 & 1.30 & 20.2 & 0.010 & 12.98 \\
\hline $0718-340$ & 3 & 1200 & 1.25 & 20.9 & 0.008 & 12.15 \\
\hline $0806-103$ & 3 & 600 & 1.20 & 20.8 & 0.007 & 15.20 \\
\hline $0915-118$ & 3 & 600 & 1.35 & 20.1 & 0.009 & 13.23 \\
\hline $0940-304$ & 3 & 600 & 1.20 & 20.7 & 0.013 & 13.12 \\
\hline $0945+076$ & 3 & 1800 & 1.23 & 20.4 & 0.010 & 15.19 \\
\hline $1002-320$ & 3 & 1200 & 1.17 & 19.9 & 0.008 & 14.52 \\
\hline $1043-290$ & 3 & 300 & 1.17 & 20.5 & 0.012 & 13.22 \\
\hline $1053-282$ & 3 & 1800 & 1.13 & 19.7 & 0.006 & 13.86 \\
\hline $1056-360$ & 3 & 1800 & 1.19 & 19.6 & 0.006 & 14.64 \\
\hline $1107-372$ & 3 & 300 & 1.2 & 19.5 & 0.005 & 9.53 \\
\hline $1123-351$ & 3 & 510 & 1.21 & 20.3 & 0.010 & 11.92 \\
\hline $1251-122$ & 3 & 600 & 1.25 & 20.1 & 0.009 & 10.63 \\
\hline $1251-289$ & 3 & 1800 & 1.17 & 18.9 & 0.004 & 12.41 \\
\hline $1257-253$ & 3 & 1200 & 1.2 & 19.8 & 0.006 & 13.92 \\
\hline $1258-321$ & 3 & 1200 & 1.10 & 19.8 & 0.015 & 10.71 \\
\hline $1318-434$ & 3 & 600 & 1.20 & 19.9 & 0.012 & 9.98 \\
\hline $1323-271$ & 3 & 1800 & 1.10 & 19.5 & 0.013 & 13.14 \\
\hline $1333-337$ & 3 & 600 & 1.20 & 19.9 & 0.006 & 9.97 \\
\hline $1344-241$ & 3 & 1200 & 1.25 & 20.4 & 0.010 & 12.37 \\
\hline $1354-251$ & 3 & 600 & 1.25 & 20.4 & 0.007 & 13.36 \\
\hline $1400-337$ & 4 & 1200 & 1.25 & 20.9 & 0.010 & 9.72 \\
\hline $1404-267$ & 4 & 1200 & 1.25 & 20.8 & 0.012 & 11.71 \\
\hline $1637-771$ & 4 & 1200 & 1.55 & 20.6 & 0.008 & 13.54 \\
\hline $1717-009$ & 4 & 1200 & 1.15 & 20.7 & 0.010 & 13.87 \\
\hline $1733-565$ & 4 & 900 & 1.25 & 20.2 & 0.008 & 15.22 \\
\hline $1928-340$ & 2 & 1200 & 1.20 & 20.2 & 0.018 & 14.31 \\
\hline $1949+023$ & 5 & 1200 & 0.72 & 20.7 & 0.010 & 14.11 \\
\hline $2221-023$ & 5 & 1200 & 0.67 & 20.9 & 0.012 & 14.46 \\
\hline
\end{tabular}


fitting) was performed using the AIAP package (Fasano 1990), which due to its high degree of interactivity, is particularly suitable in analyzing the morphology of galaxies embedded in such high density regions.

The problem of obtaining reliable surface photometry of dumbbell systems was faced by adopting the two-galaxy fitting strategy outlined in Paper I, which allows us to fully separate the two galaxies. Contour plots for all dumbbell systems, together with those of the two members are shown in Fig. 2.

From this analysis we derived photometric and structural parameters (surface brightness, ellipticity, position angle and Fourier coefficients) as a function of the equivalent radius $r=a \times(1-\epsilon)^{1 / 2}$ where $a$ is the semimajor axis and $\epsilon$ is the ellipticity of the ellipse fitting a given isophote. Isophotes can not be fitted in the innermost few arcsec of the galaxy because of the small number of pixels involved. To cope with this limitation, we extracted an azimuthally averaged radial profile, centered on the center of the first useful isophote. If the nucleus was saturated, the short exposure was used. The agreement between this average radial profile and that obtained from isophote fitting was always excellent in the common region, thus the two profiles were joined smoothly to fully model the core and the outer region of the galaxies. In the following analysis we consider this combined profile as the final luminosity profile.

After fitting of the isophotes with ellipses, photometric and morphological profiles have been obtained according to the procedure described in Paper I. For each galaxy, the luminosity radial profile, the major axis position angle (defined from North to East), the ellipticity, and $c 4$ coefficient profiles as a function of the semi-major axis are shown in Fig. 3. The Fourier coefficient $c 4$ measures the deviation of the isophotes from the best fitting ellipse. A positive values indicate the isophote is excessively elongated along the major axis, i.e., it is similar to a disk, while a negative $c 4$ means the isophote is boxy.

The residual background variations inside each frame were used to derive, according to Fasano \& Bonoli (1990), proper errors for morphological and photometric parameters.

For the dumbbell system in Fig. 3 we show only the luminosity profile of the radio source.

\section{Comparison with previous results}

Several objects presented here were previously studied by Lilly \& Prestage (1987, LP87) and Smith \& Heckman (1989a,b, SH89). In common between our and LP87 samples there are 13 objects $(0255+058,0325+023,0427-539$, $0430+052,0453-206,0620-526,0625-354,0625-536$, $0915-118,0945+076,1318-434,1333-337$, and $2221-$ 023), while eight are in common with SH89 (0255+ 058, $0325+023,0430+052,0945+076,1251-122,1717-009$, $1949+023$, and $2221-023)$.
LP87 give Cousins metric $R$ magnitudes for a fixed aperture of $19.2 \mathrm{kpc}$ (for $H_{0}=50 \mathrm{~km} \mathrm{~s}^{-1} \mathrm{Mpc}^{-1}$ ), whereas SH89 report $V$ and $B$ bands isophotal $\left(m_{25}\right)$ magnitudes. In order to perform an external check on our photometry, we derived metric $R$ magnitudes at $19.2 \mathrm{kpc}$ and isophotal magnitudes $V_{25}$ (assuming $V-R=0.6$ as appropriate for low redshift elliptical galaxies) for the common objects (Fig. 4), finding on average:

$$
\begin{aligned}
& <\Delta R_{19.2}>_{\mathrm{LP} 87}=-0.12 \mathrm{mag} \quad(\mathrm{rms}=0.29) \\
& <\Delta V_{25}>_{\mathrm{SH} 89}=0.02 \mathrm{mag} \quad(\mathrm{rms}=0.36) .
\end{aligned}
$$

It is worth noticing that $0255+058$ and $1251-122$ are dumbbell galaxies, while a bright, edge-on spiral galaxy projects on-top of $1318-434$. The measure of the luminosity of these galaxies is therefore particularly difficult and dependent on the details of the adopted measuring strategy. Not surprisingly $0255+058$ is the object with the largest discrepancy with respect to SH89.

If we remove this object, the scatter becomes 0.27 and 0.19 for the comparison with LP87 and SH89, respectively. As a whole, our photometry agrees on average with previous photometry within $\sim 0.1$ magnitudes.

\section{Conclusions}

We presented surface photometry analysis for 50 radio galaxies which complement our previous study of 29 objects from a complete sample of 95 low redshift radio galaxies. Detailed morphological and photometrical properties of the galaxies are reported. As previously found by other studies (e.g., SH89; Ledlow \& Owen 1995), we confirm that the galaxies associated with this kind of radio emission have mostly elliptical morphology.

In some cases, however, the galaxy hosting the radio source is found to have a substantial disc component (S0like). Moreover the surface brightness radial profiles of radio galaxies often exhibit deviations from de Vaucouleurs $r^{1 / 4}$ profile, due to the presence of nuclear point sources (likely associated with the active nucleus) and/or to low surface brightness extended halos.

A detailed description of each object is given in the appendix, while a full discussion of the results for the whole observed sample of 79 radio galaxies is presented in Govoni et al. (1999).

Acknowledgements. This work was partly supported by the Italian Ministry for University and Research (MURST) under grant Cofin98-02-32, and has made use of the NASA/IPAC Extragalactic Database (NED) which is operated by the Jet Propulsion Laboratory, California Institute of Technology, under contract with the National Aeronautics and Space Administration.

\section{Appendix A: Results for individual objects}

0255 + 058: The optical counterpart of $3 \mathrm{C} 75$, the central radio source of the cluster Abell 400, is an interesting 

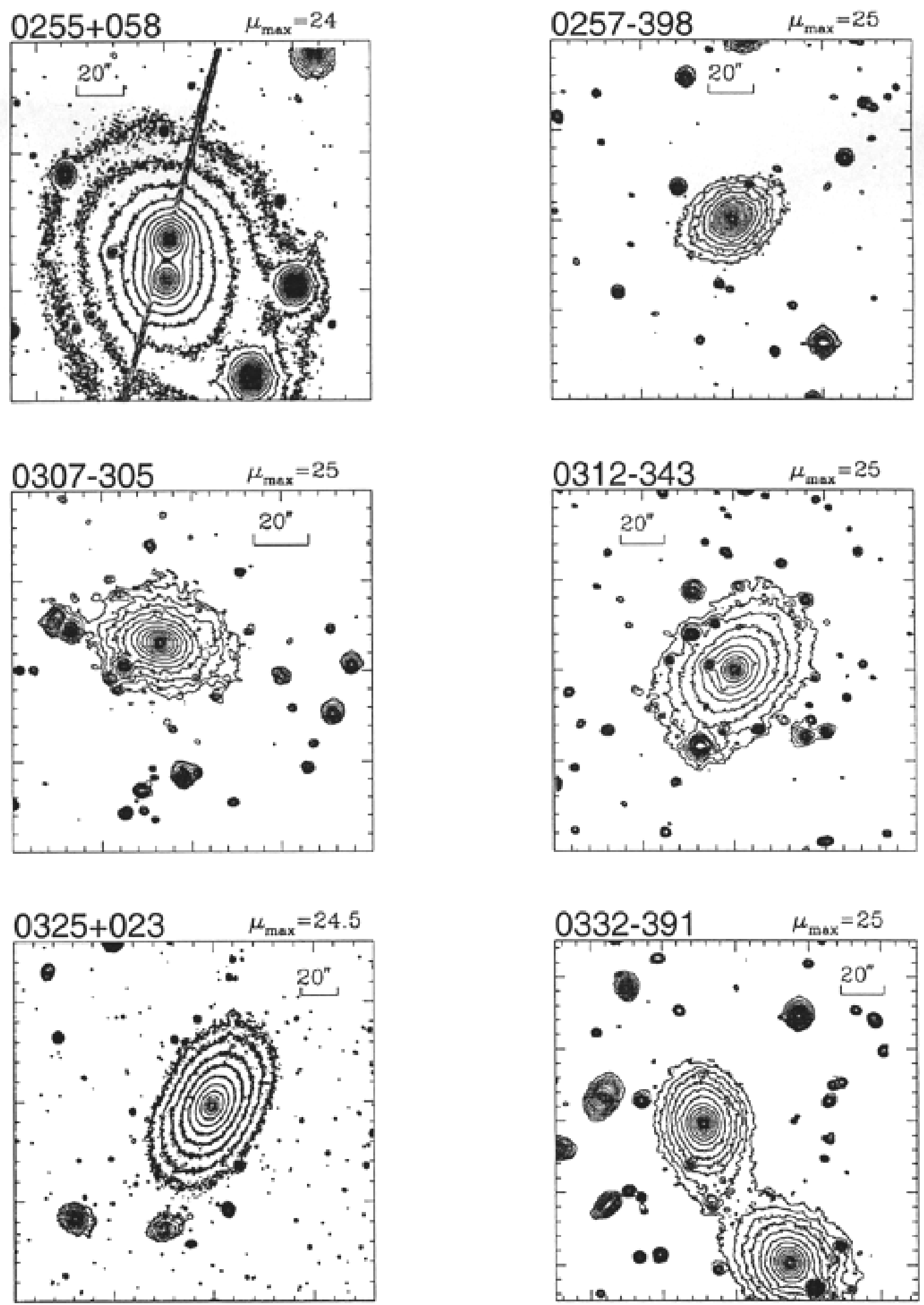

Fig. 1. Isophotal contour plots of the central portion of the Cousins $R$ band CCD image of selected sources. The optical counterpart of the radio source is always placed at the center of the figure. The image scale is indicated; North is up and East to the left. Contours start at the surface brightness indicated on top of each panel, and are separated by $0.5 \mathrm{mag} / \mathrm{arcsec}^{2}$ 

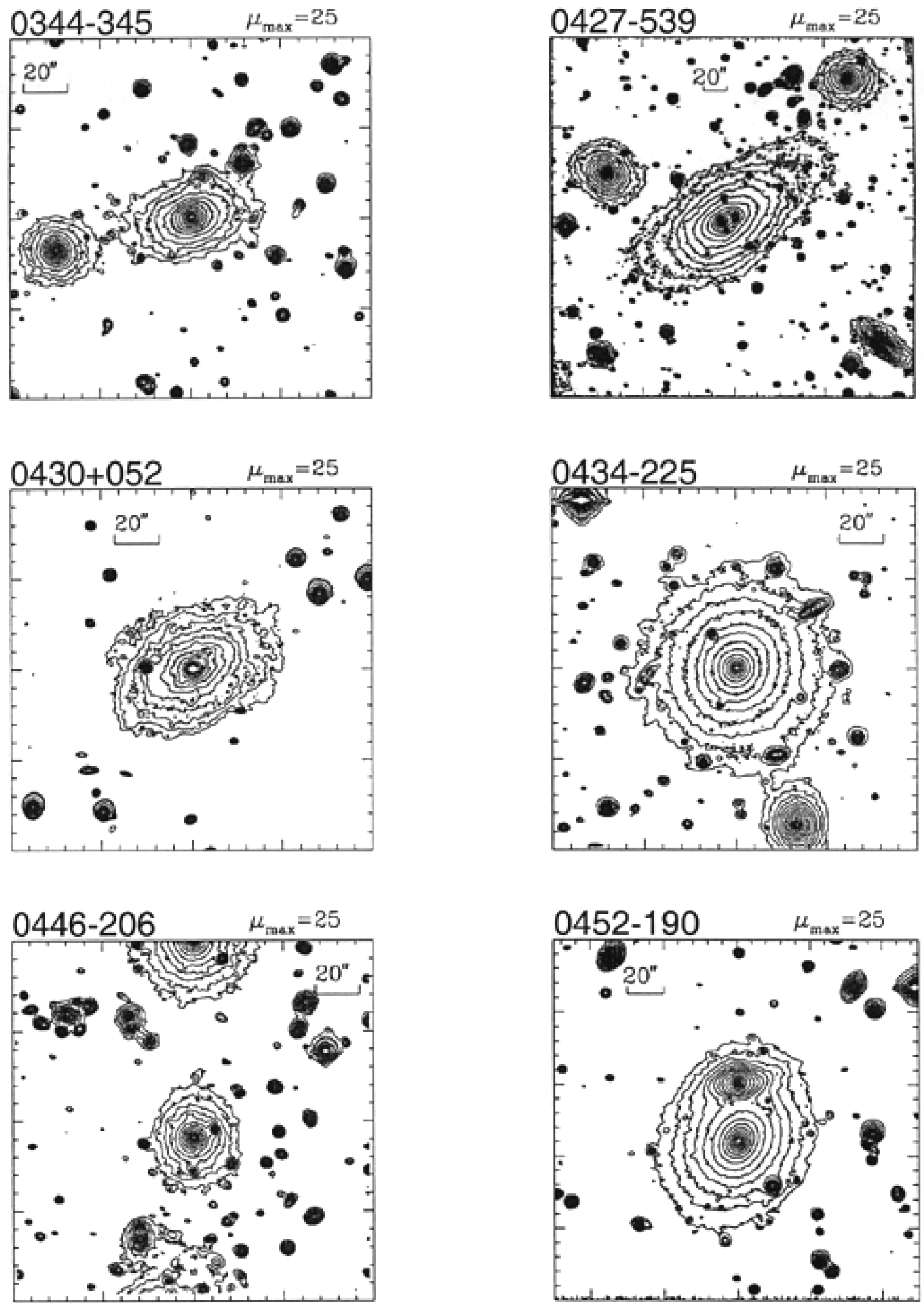

Fig. 1. continued 

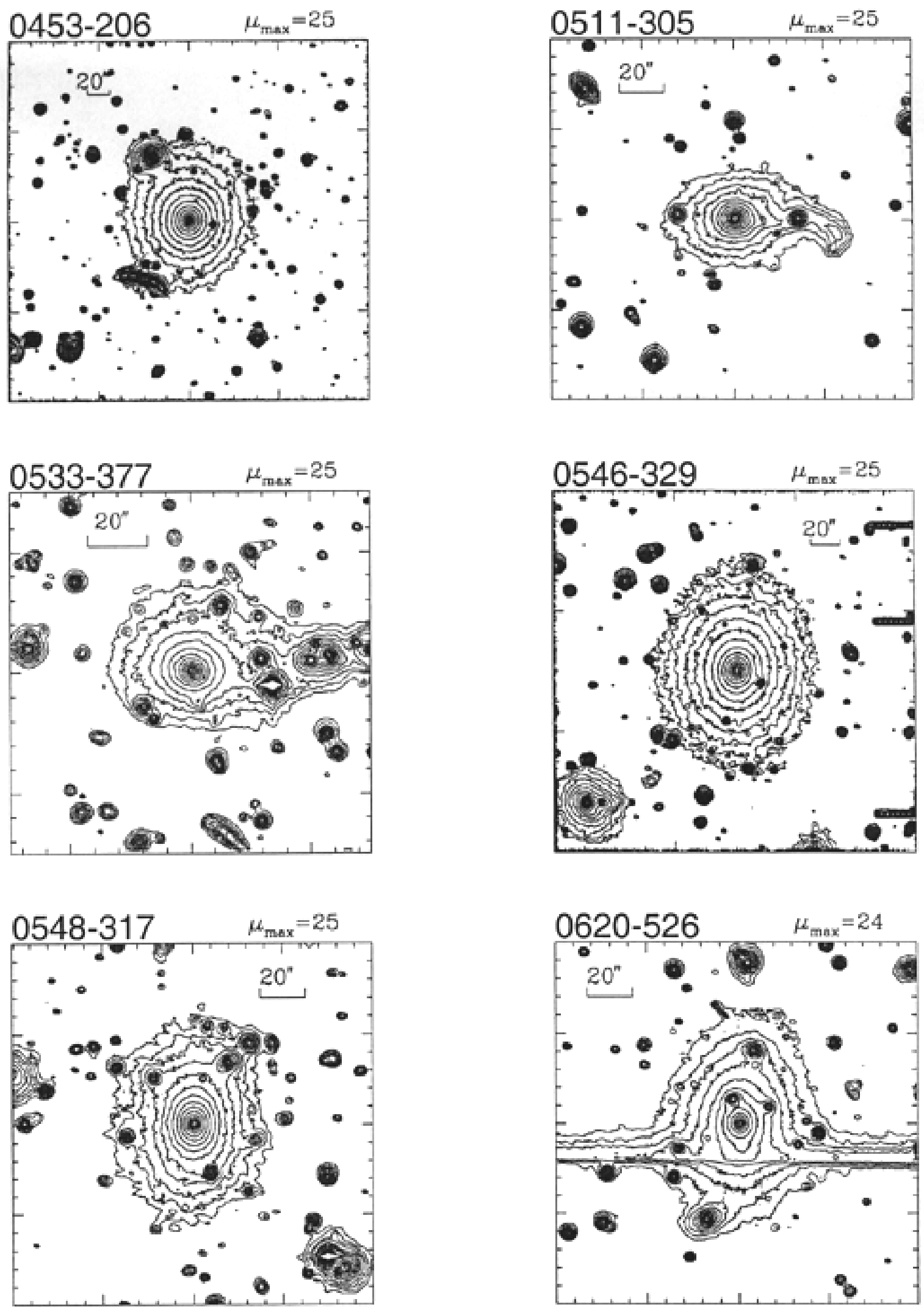

Fig. 1. continued 

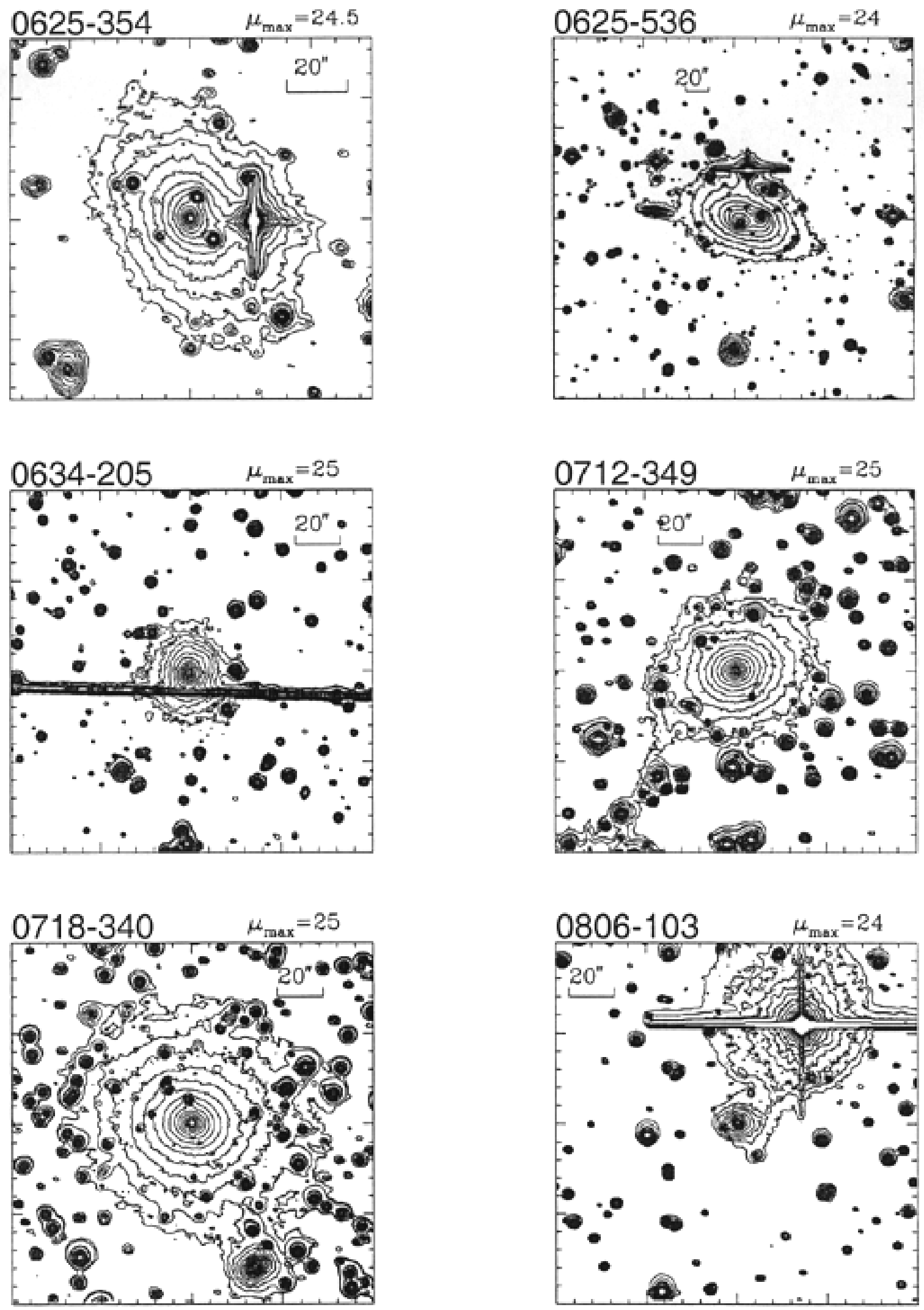

Fig. 1. continued 

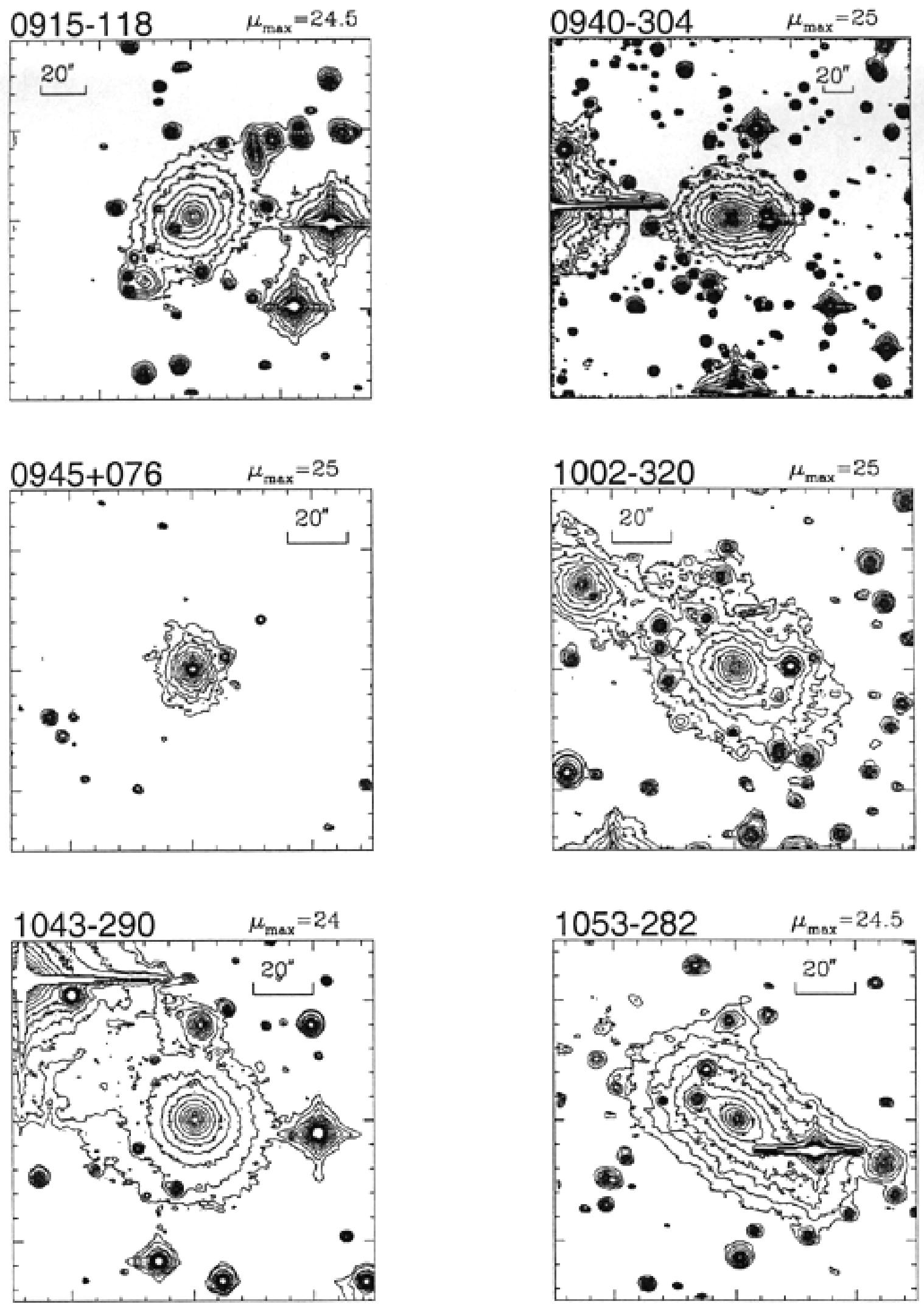

Fig. 1. continued 

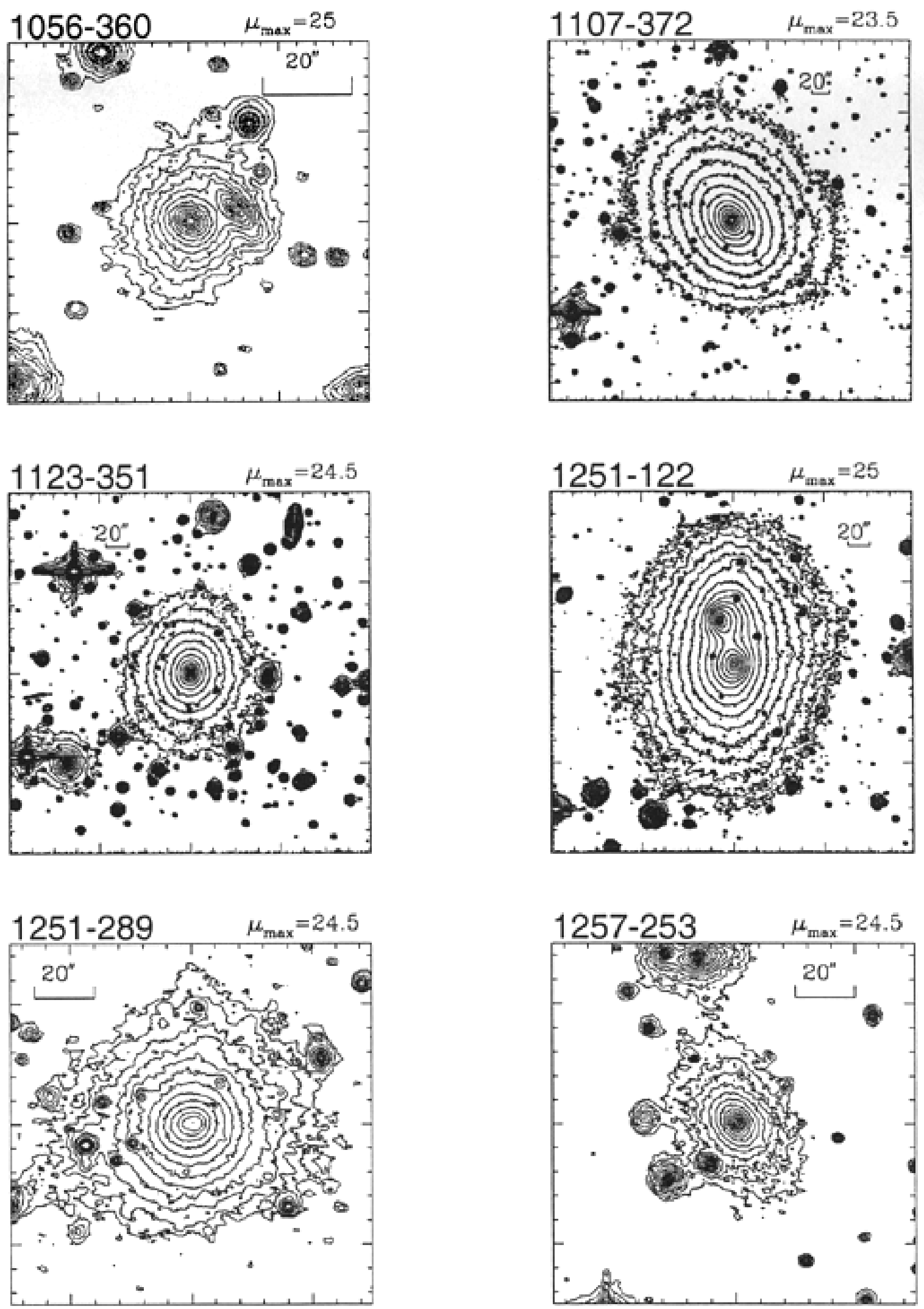

Fig. 1. continued 

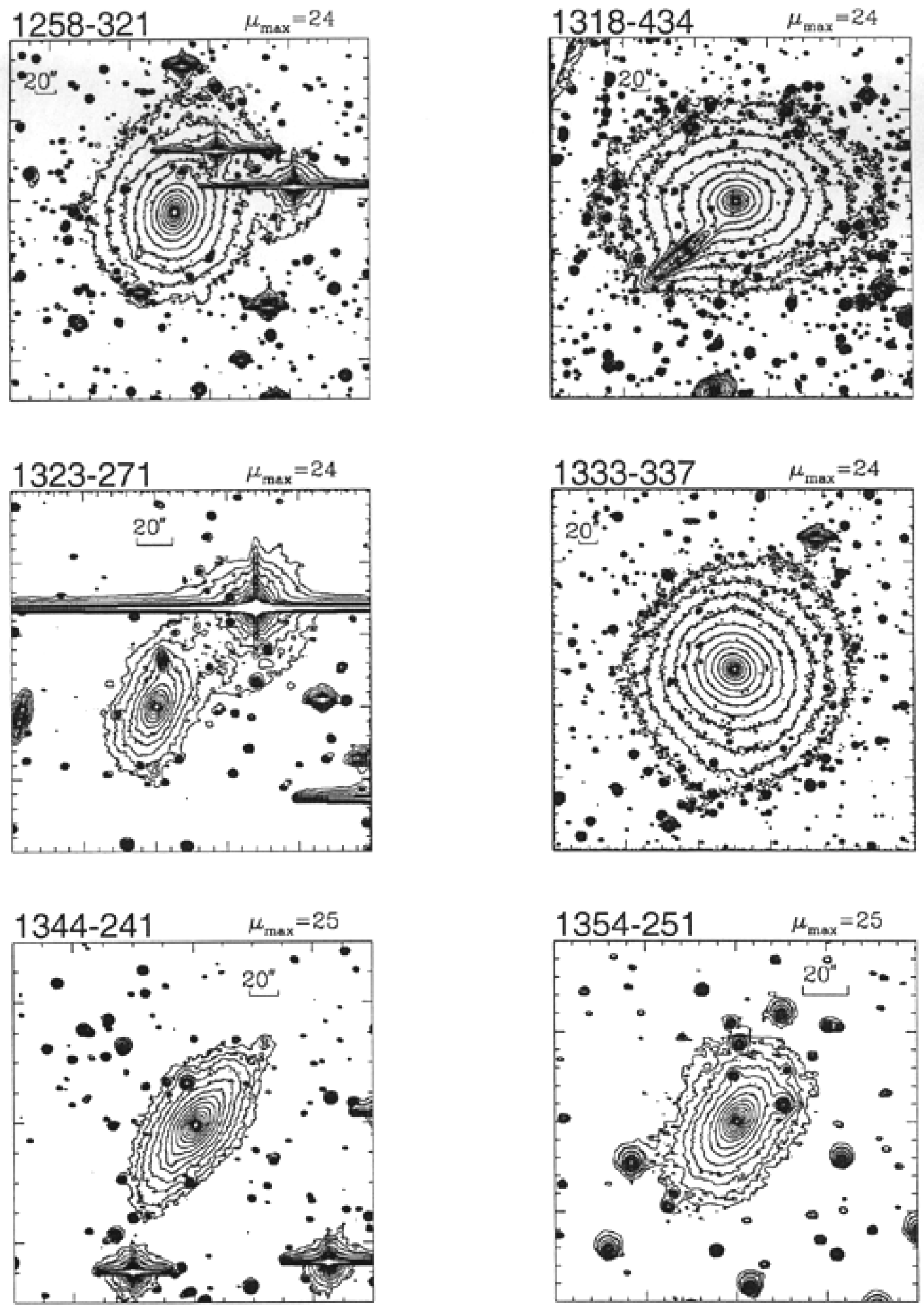

Fig. 1. continued 

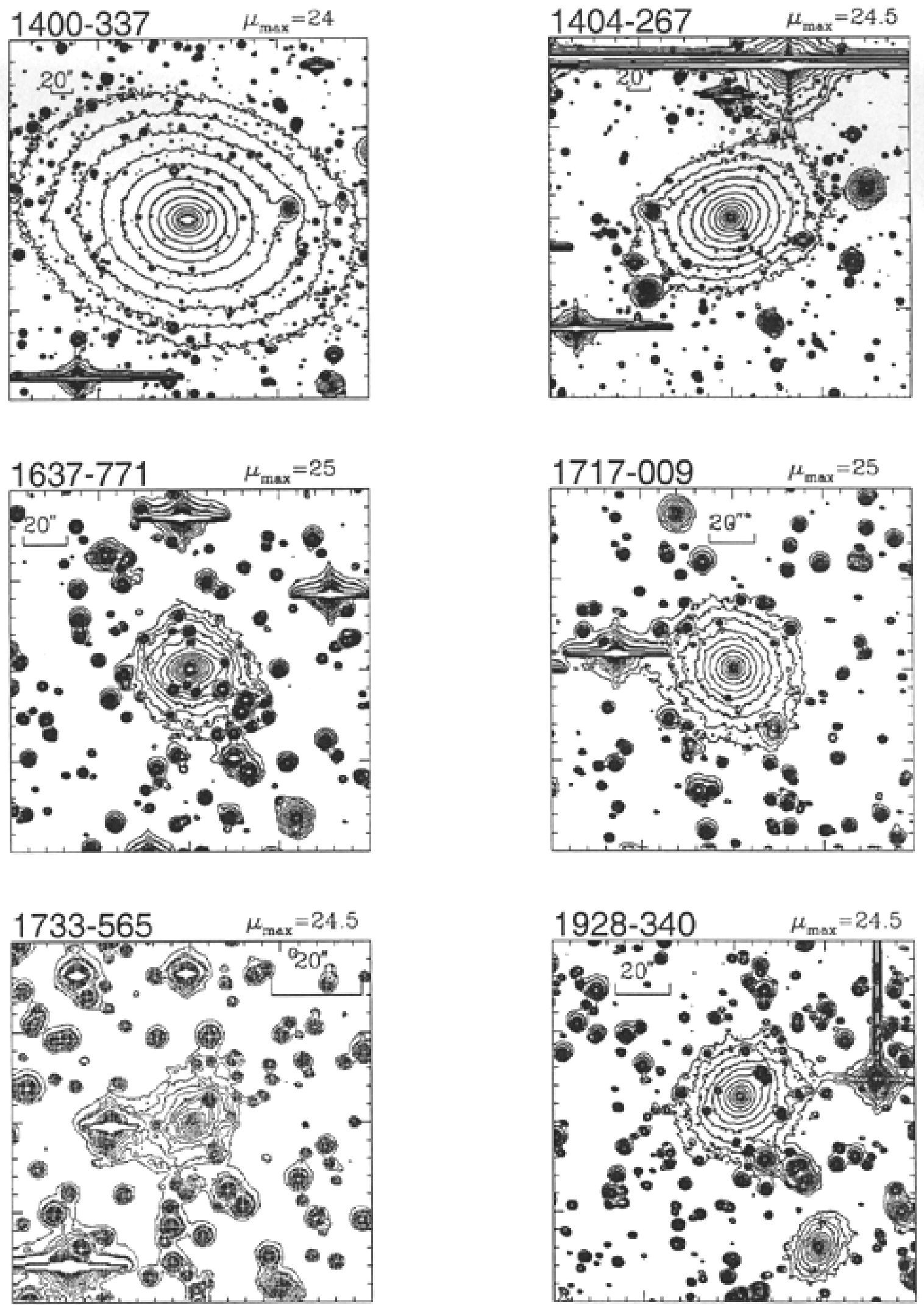

Fig. 1. continued 


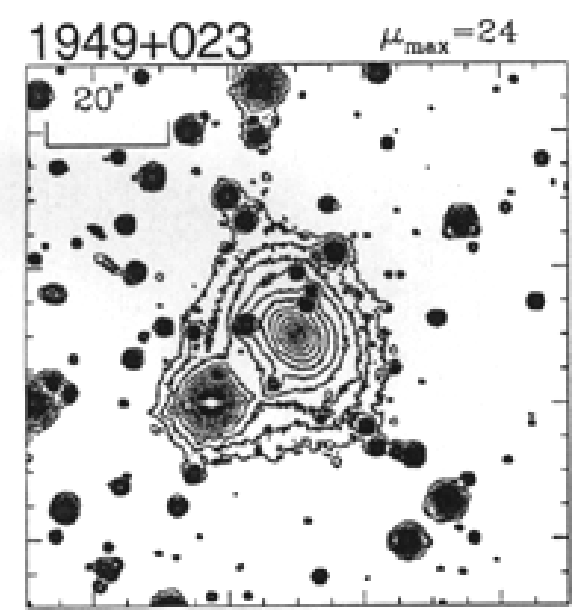

Fig. 1. continued

case of a dumbbell galaxy, with components separated by $\sim 20^{\prime \prime}$ and with a radial velocity difference of $500 \mathrm{~km} \mathrm{~s}^{-1}$. Twin radio jets depart from each of the two optical nuclei (Owen et al. 1985), making this radio source extremely unusual. The large scale radio structure is classified as FRI (Morganti et al. 1993).

Unfortunately, a bright satellite crossed the field, passing close to the central part of the dumbbell system (see Fig. 1). Nevertheless, using the AIAP masking facility, we were able to obtain a photometric deblending, by iterative modeling of the two components. In spite of the appearance, the geometry of the two galaxies looks rather regular, apart from a slight off-centering of the outermost isophotes. Luminosity and geometrical profiles suggest both components are ellipticals. In particular, the luminosity profile (see Fig. 3) of the northern galaxy is consistent with the presence of a nuclear point source.

0257 - 398: The optical counterpart of this radio source is a rather isolated galaxy at $z \simeq 0.066$ (Scarpa et al. 1996). The geometrical profiles of this object are highly suggestive of an S0 morphology (increasing ellipticity, positive $c 4$ coefficient).

$0307-305$ : This object seems to be a rather isolated galaxy. Its luminosity and geometrical profiles suggest a regular elliptical morphology, with a possible nuclear point source.

0312 - 343: The source looks like a large elliptical with regular morphology. The luminosity profile strongly suggests the presence of a bright nuclear point source.

$\mathbf{0 3 2 5}+\mathbf{0 2 3}$ : The radio morphology of $3 \mathrm{C} 88$ is dominated by two symmetric and well developed lobes at $\mathrm{PA}=60^{\circ}$, and is classified as FRII (Morganti et al. 1993). The optical counterpart which coincides with the radio nucleus, is an elliptical galaxy, whose major axis has position angle $\mathrm{PA}=-30^{\circ}$. This is remarkably well defined and stable (Fig. 3) and, within the uncertainty, orthogonal to the radio structure.

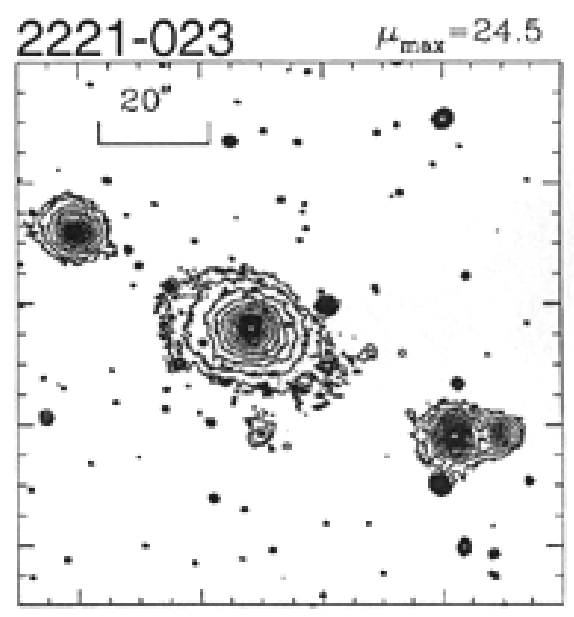

0332 - 391: A radio map of this FRI radio source is reported by Jones \& McAdam (1992). Figure 1 shows that the host galaxy is embedded in a quite dense environment. In particular, it seems to interact with another (very similar) galaxy, whose angular separation from the radio galaxy is $\sim 76^{\prime \prime}$. The luminosity profile is consistent with the presence of a nuclear point source.

0344 - 345: As for the previous case, the galaxy hosting this radio source turns out to be embedded in a rich environment and seems to interact with a close elliptical companion. The luminosity profile is consistent with the presence of a nuclear point source, whereas the geometrical profiles suggest regular elliptical morphology. This source is also interesting for having strong optical emission lines (Scarpa et al. 1996), and complex radio morphology (Jones \& McAdam 1992).

0427 - 539: The optical counterpart of this radio source is a spectacular case of dumbbell morphology in rich environment, with nuclei separated by $\sim 30^{\prime \prime}(\sim 33 \mathrm{kpc})$. The radio source is associated with the South-East component of the dumbbell system. After deblending the two galaxies, we observe a regular elliptical morphology for the brightest object, whereas the other galaxy shows a rather amorphous and broad light distribution (see contours in Fig. 2).

$0430+$ 052: $3 \mathrm{C} 120$ is a well studied radio source, displaying superluminal motion (Zensus 1989). The spectrum of the optical counterpart is quasar like (Tadhunter et al. 1993), and the galaxy has been often classified as Seyfert 1, even if its spiral morphology has never been clearly established.

0434 - 225: The galaxy hosting this radio source is embedded in a moderately rich environment. It has regular elliptical morphology, as illustrated by the luminosity and geometrical profiles, as well as by its isophotal contours. The luminosity profile is consistent with the presence of a nuclear point source. 

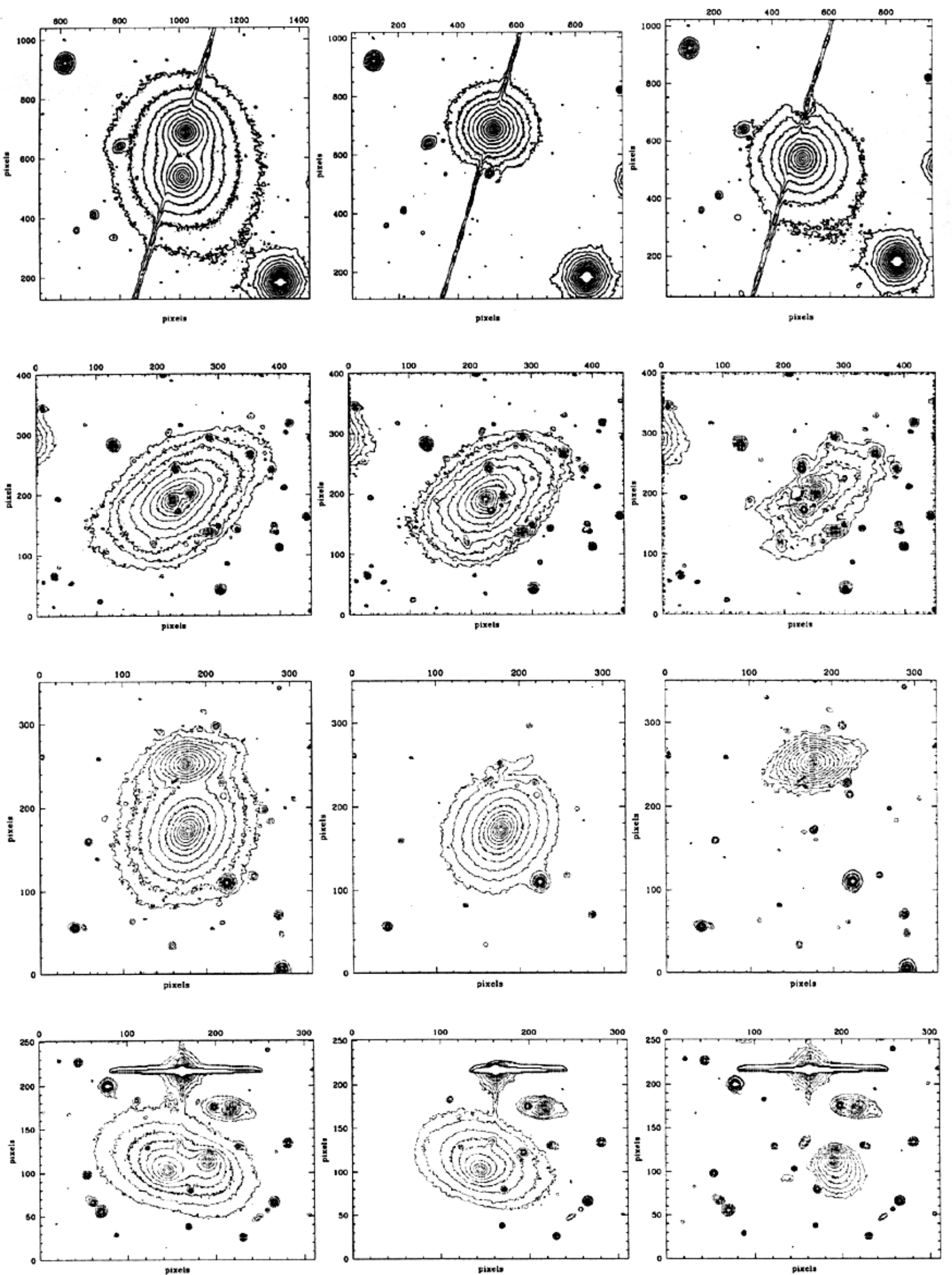

Fig. 2. Contour plots for all dumbbell systems, together with those of the two members as obtained after they have been separated using a two - galaxy fitting procedure (Paper I). Objects are, from top to bottom, 0255+058, 0427 - 539, 0452 - 190, $0625-536$, and $1251-122$ 

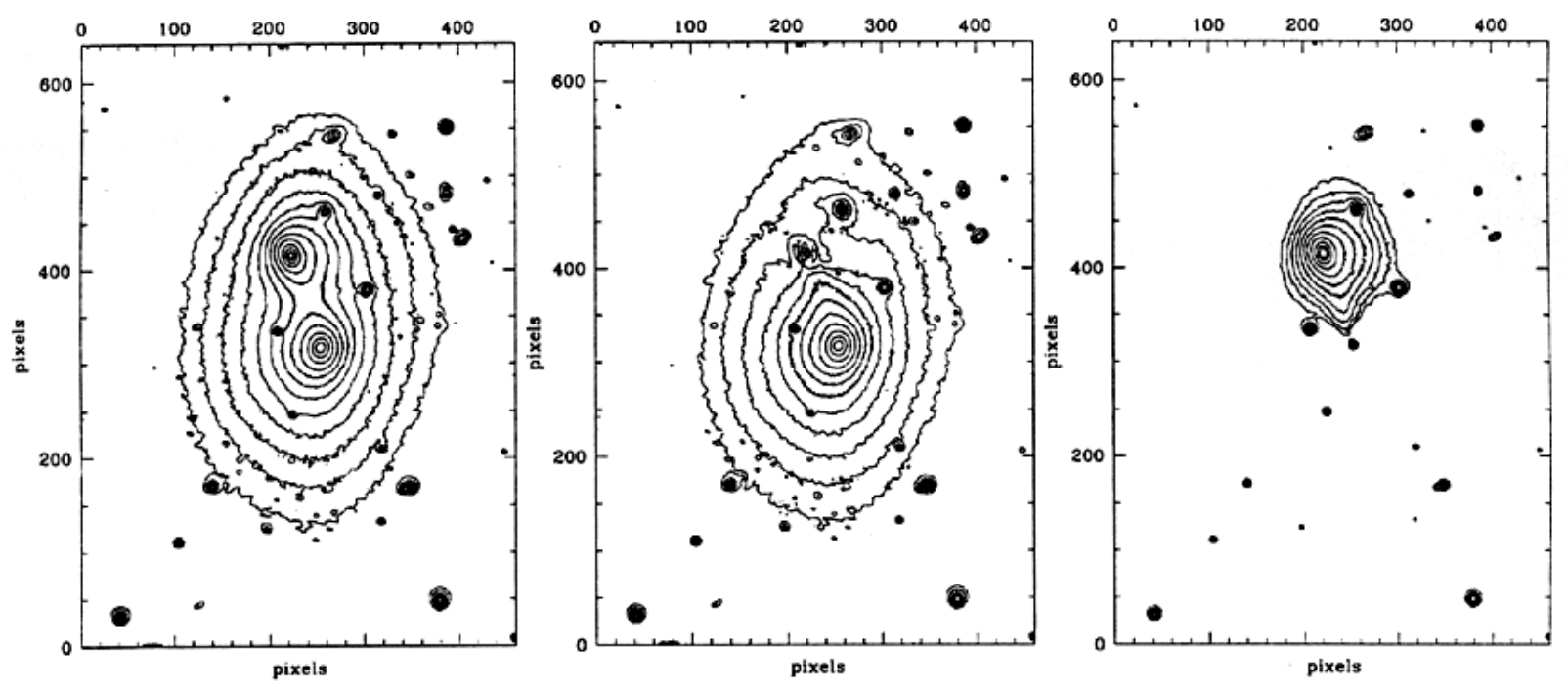

Fig. 2. continued

0446 - 206: The optical counterpart of this radio source lies in the very dense environment of the cluster Abell 514 . The host galaxy is a normal elliptical.

0452 - 190: An interesting case of dumbbell galaxy. The radio source coincides with the Southernmost component. After deblending, both galaxies show very regular morphology (see Fig. 2), suggesting the dumbbell appearance may just be due to chance projection. Based on the shape of their radial profile we conclude the northernmost galaxy is an S0, and the other one an elliptical.

0453 - 206: In the optical band NGC 1692 looks like a large, undisturbed elliptical galaxy, in spite of the presence of two nearby galaxies both at a projected distance of $\sim 66 . " 5$ from the radio galaxy. The south-east companion is likely to be an edge-on spiral showing a pronounced C-shape.

0511 - 305: Even if the optical counterpart of this radio source is located in a rather poor environment, the host galaxy appears morphologically disturbed by the presence of some small companions. In particular, two small compact galaxies on opposite sides with respect to the galaxy center are aligned with an elongated structure extending for $\sim 30^{\prime \prime}$ westward.

0533 - 377: The optical counterpart of this radio source is an elliptical galaxy, located at the end of a chain of small galaxies. The surrounding environment is very dense, with several galaxies superposed on the main object.

0546 - 329: The host galaxy of this radio source is a large, normal elliptical. The only noticeable thing being the presence of a nearby galaxy pair.

0548 - 317: A rather normal elliptical galaxy in a rich environment, with several small galaxies projecting on its halo. The luminosity profile suggests the existence of a nuclear point source.
0620 - 526: This FRI radio source (Jones \& McAdam 1992) shows weak emission lines superposed onto the continuum spectrum of a typical early-type galaxy (Simpson et al. 1996). The source was also detected in the X-ray band (Gioia \& Luppino 1994). Unfortunately, our image of the galaxy is disturbed by the presence of several saturated columns of the CCD, due to a nearby bright star. Nevertheless, using appropriate masking we were able to produce a reliable radial profile, from which we infer the existence of a nuclear point source.

0625 - 354: The optical counterpart of this FRI radio source, located at the center of the cluster Abell 3392, is a giant elliptical embedded in a rich environment. It is worth noticing the presence of a strong point source in the nucleus of this galaxy, also supported by the emission lines observed in its optical spectrum (Tadhunter et al. 1993).

0625 - 536: The optical counterpart of this radio source is the South-East member of a dumbbell system, located in the cluster Abell 3391. After deblending the two galaxies with the iterative two-galaxy fitting procedure (Fig. 2), we found both galaxies show strong displacement of the isophotal centers roughly perpendicular to their alignment, as expected in strong interactions.

0634 - 205: The host galaxy is a normal elliptical lying in a rather poor environment. Strong emission lines have been observed in its optical spectrum (Simpson et al. 1996). The image of the galaxy is disturbed by the light from a satellite which passed close to the center of the source. This structure was masked during isophotal analysis.

0712 - 349: The galaxy hosting this radio source is a normal elliptical with regular morphology in a poor environment. 

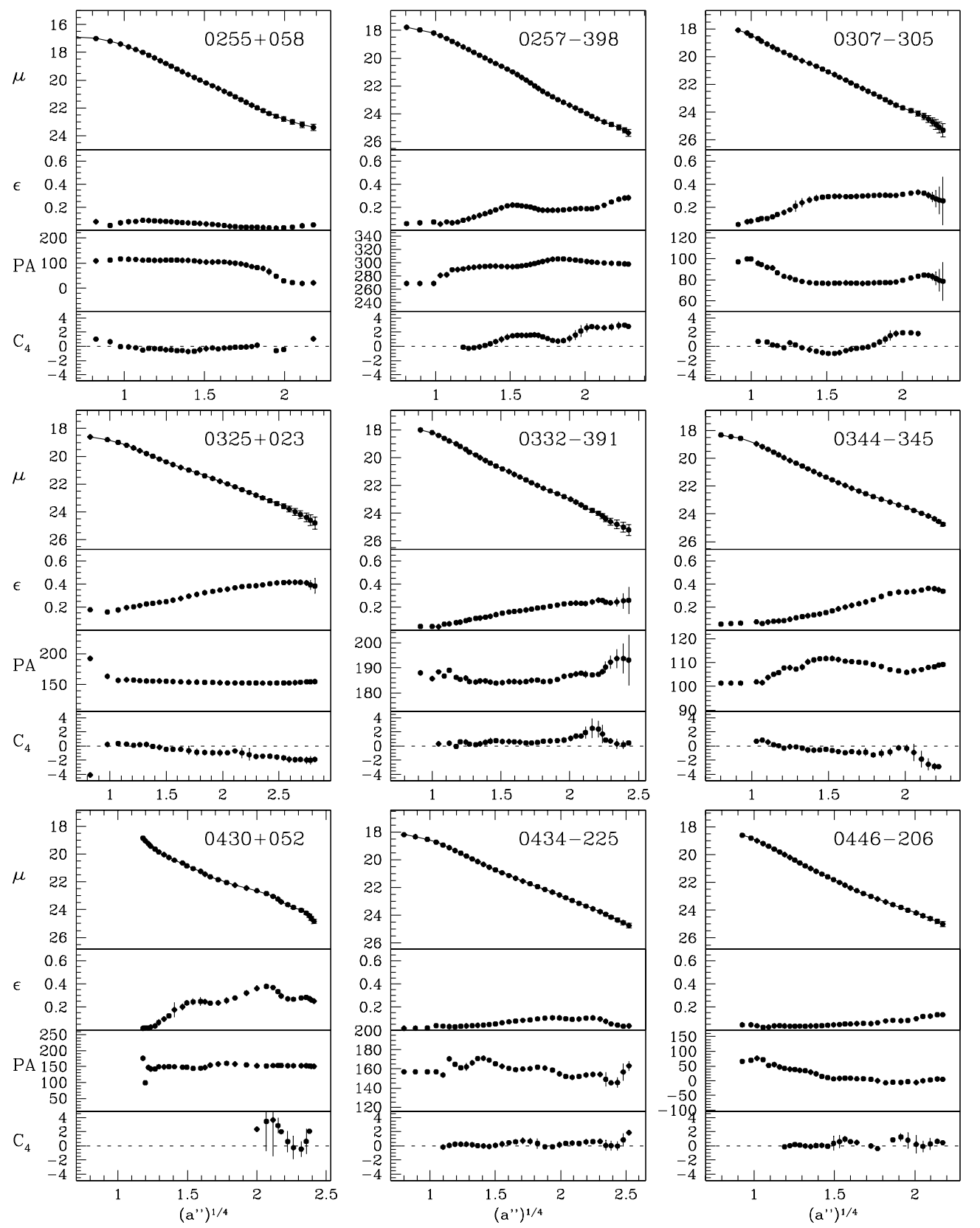
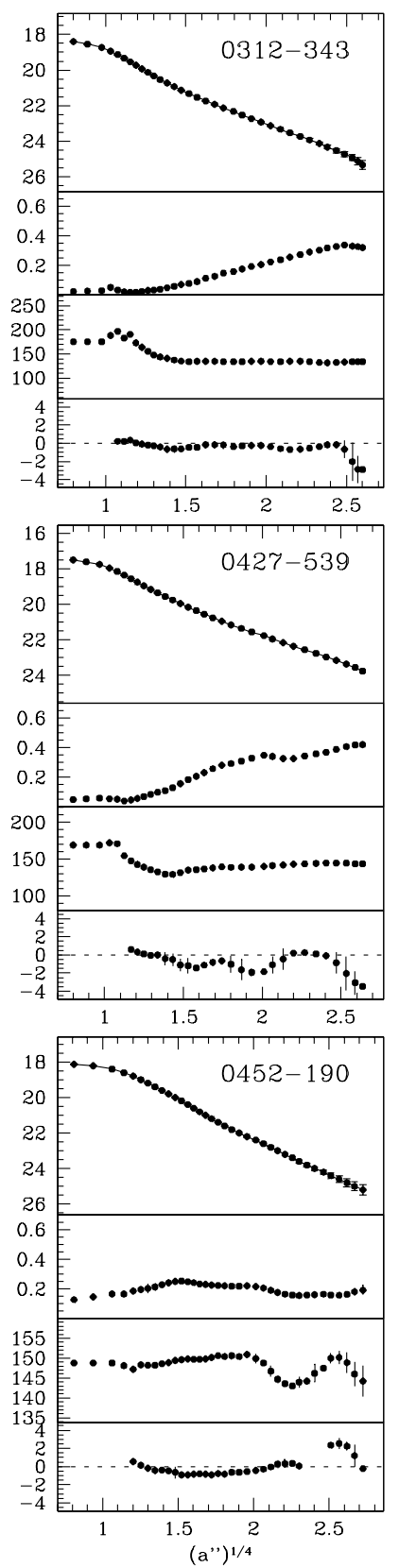

Fig. 3. Morphological and photometric profiles as a function of the semimajor axis $a^{1 / 4}$ (arcsec). From top to bottom we report: surface brightness $\mu$ (mag/arcsec $\left.{ }^{2}\right)$; ellipticity $\epsilon$; major axis position angle PA in degree from North to East; Fourier coefficients $c 4$. When not visible, the $1 \sigma$ error bars are smaller than plotted symbol

0718 - 340: The optical counterpart looks like a normal elliptical with regular morphology in a poor environment.

0806 - 103: 3C 195 is an FRII radio source (Morganti et al. 1993), with the optical counterpart exhibiting emission lines in its optical spectrum (di Serego Alighieri et al. 1994). The host galaxy is located nearby a very bright star which makes it difficult to derive luminosity andgeometrical profiles extended to the outer regions. The brighter part of the galaxy, which can be reliably studied, suggests this galaxy has complex structure and a nuclear point source. The environment is relatively rich and at least two small galaxies may be gravitationally interacting with the radio galaxy.

0915 - 118: The optical counterpart of the FRI radio source 3C 218 (Morganti et al. 1993), is an earlytype galaxy most likely a member of a small group. 

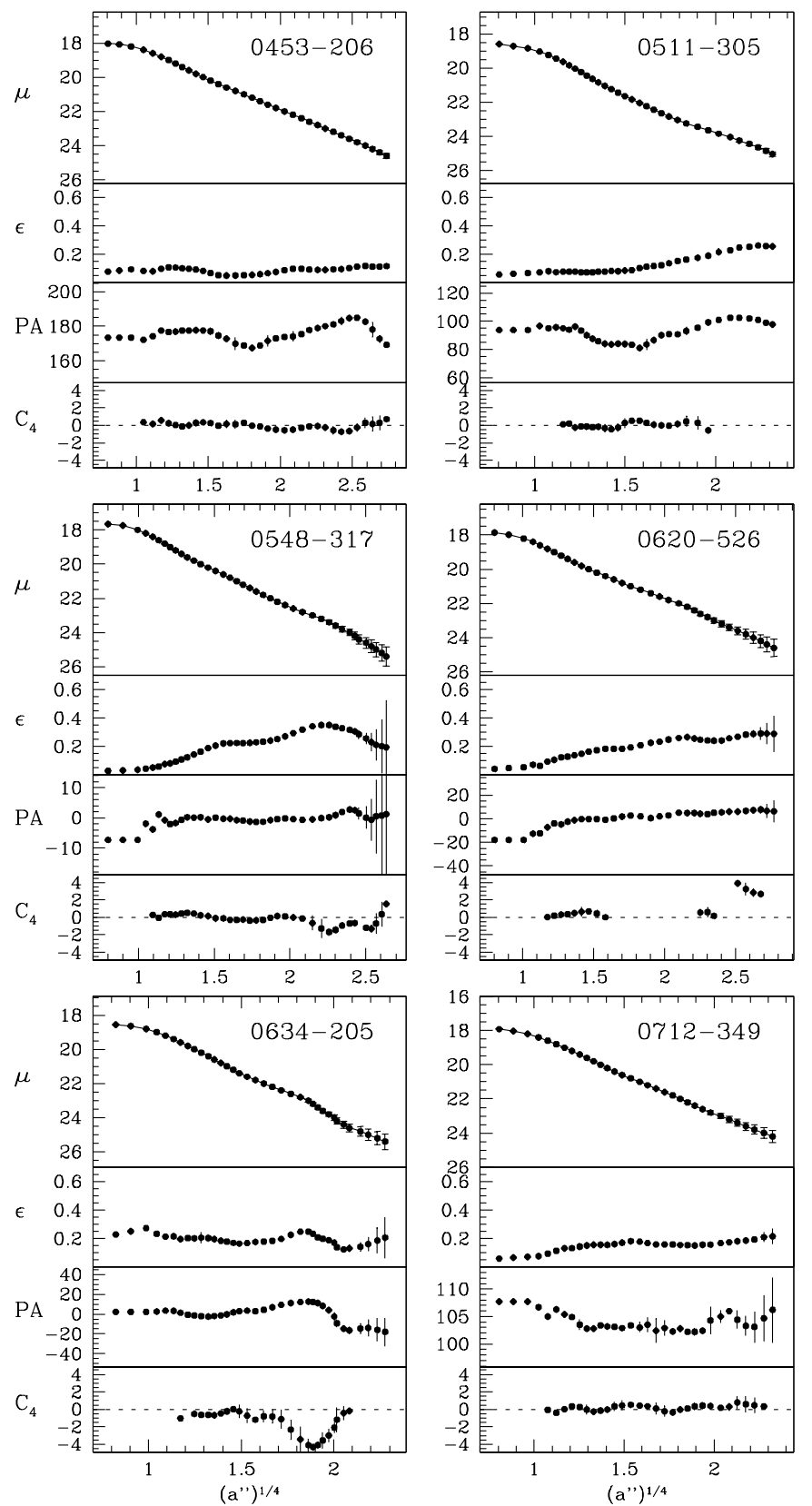

Fig. 3. continued

Ionization emission lines were detected in its optical spectrum (Simpson et al. 1996).

0940 - 304: The optical spectrum of this radio source is of an early-type galaxy with old stellar population (Scarpa et al. 1996). The optical morphology confirms this classification. The environment is poor.

$\mathbf{0 9 4 5}+\mathbf{0 7 6}$ : The radio morphology of $3 \mathrm{C} 227$ is very elongated East-West with terminal hot spots, and is classified as FRII (Morganti et al. 1993). The optical counterpart resides in a poor environment, and apart from the slight tendency to have disky isophotes, the most
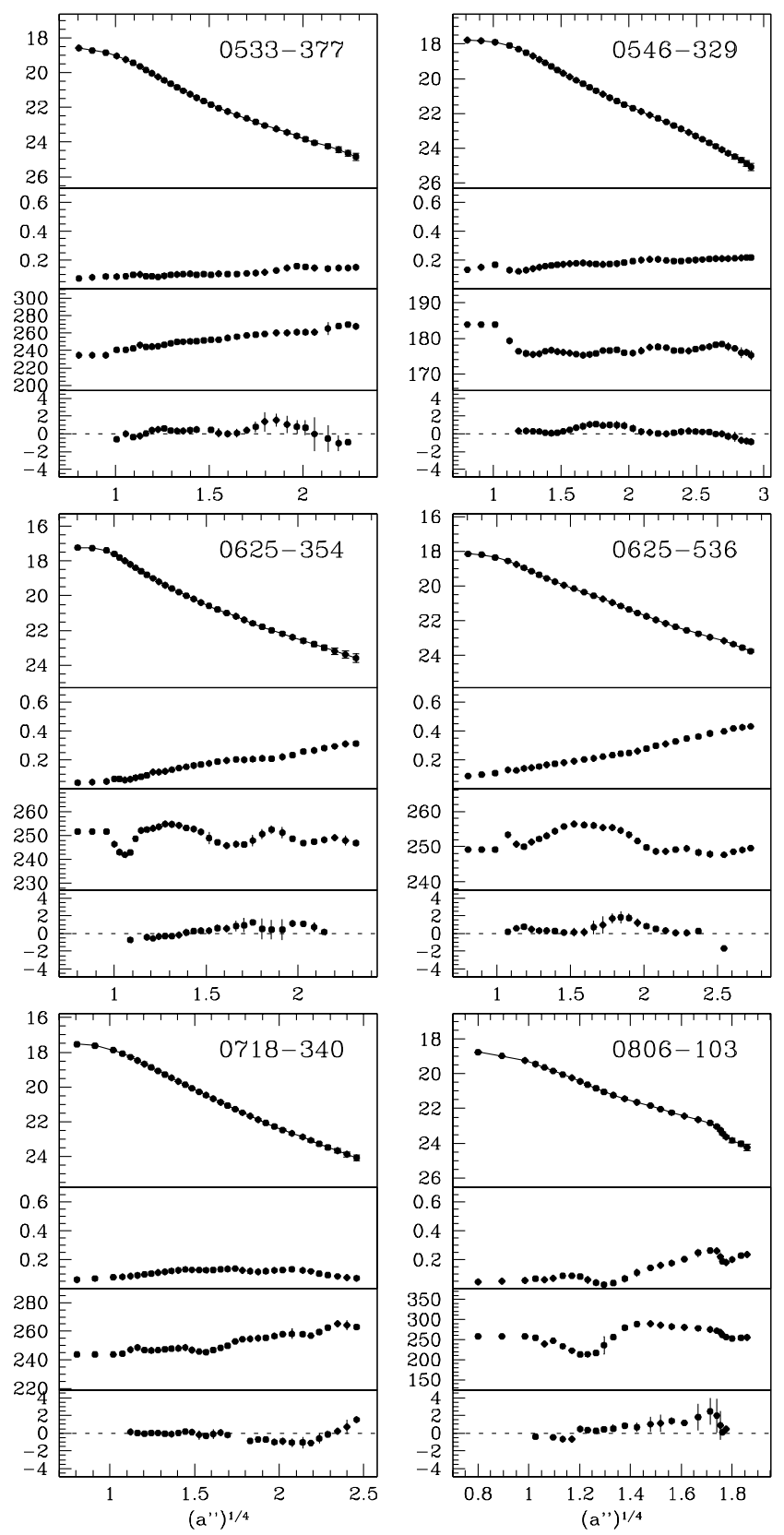

important optical feature is the presence of a very bright nuclear source. This is consistent with the detection of strong emission lines with broad wings in the optical spectrum (Simpson et al. 1996). The major axis of the galaxy, at almost constant position angle $\mathrm{PA} \sim 0^{\circ}$, is perpendicular to the radio structure.

1002 - 320: The optical counterpart is an elliptical galaxy, most likely interacting with a nearby companion at projected distance $\sim 56^{\prime \prime}$.

1043 - 290: Its optical spectrum is characteristic of an elliptical galaxy (Scarpa et al. 1996). The surface 

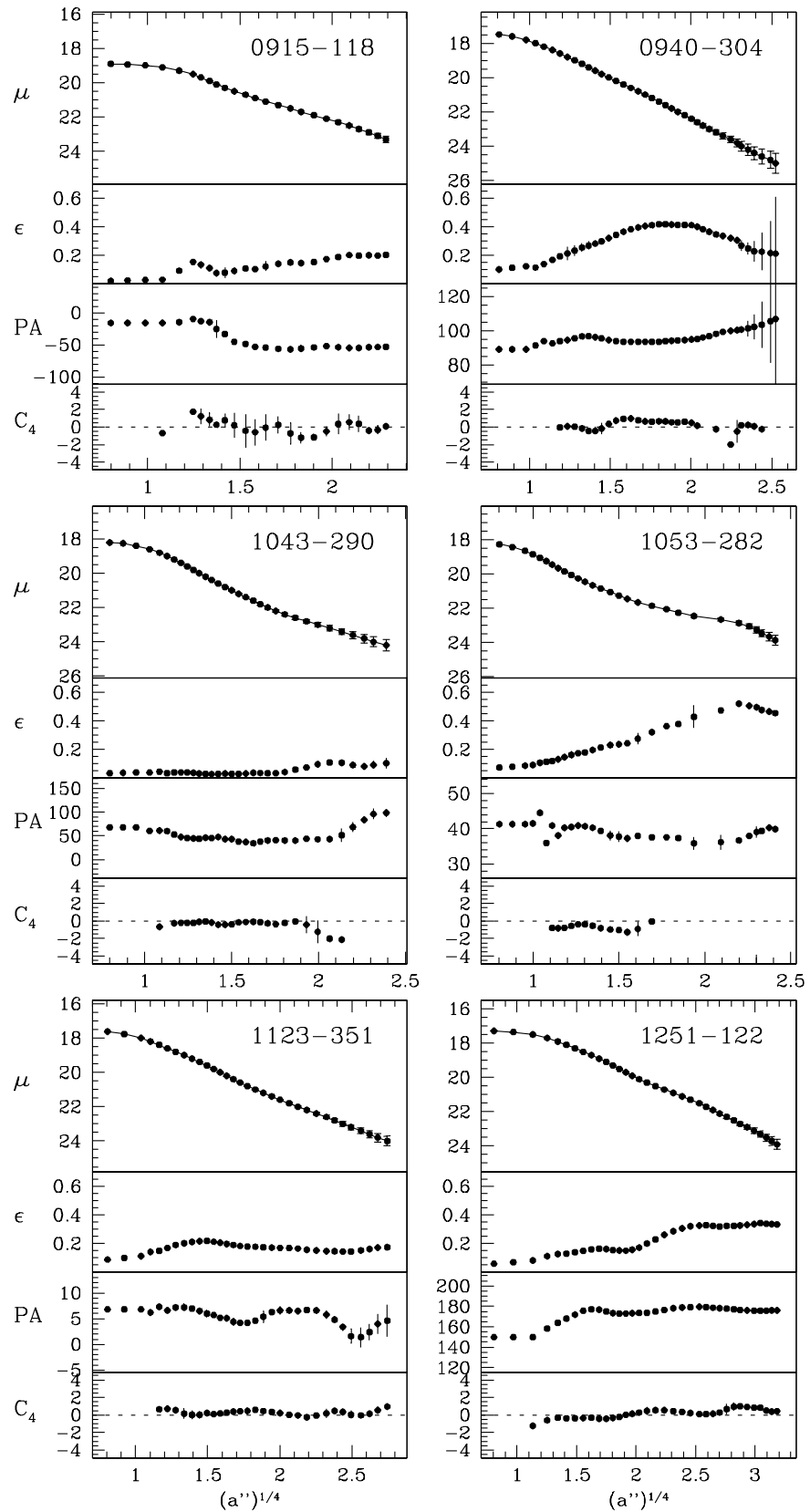

Fig. 3. continued

photometry of this galaxy is perturbed by the influence of a bright nearby star. Its morphology is clearly of elliptical type and its luminosity profile is reliable enough to indicate the presence of an outer halo. The environment looks relatively rich.

1053 - 282: This object lies in rich environment and is surrounded by several small galaxies, some of which are likely to be in interaction with the radio source. The surface photometry is made difficult both because of the above mentioned companions and also due to the presence of a relatively bright star projecting onto the galaxy
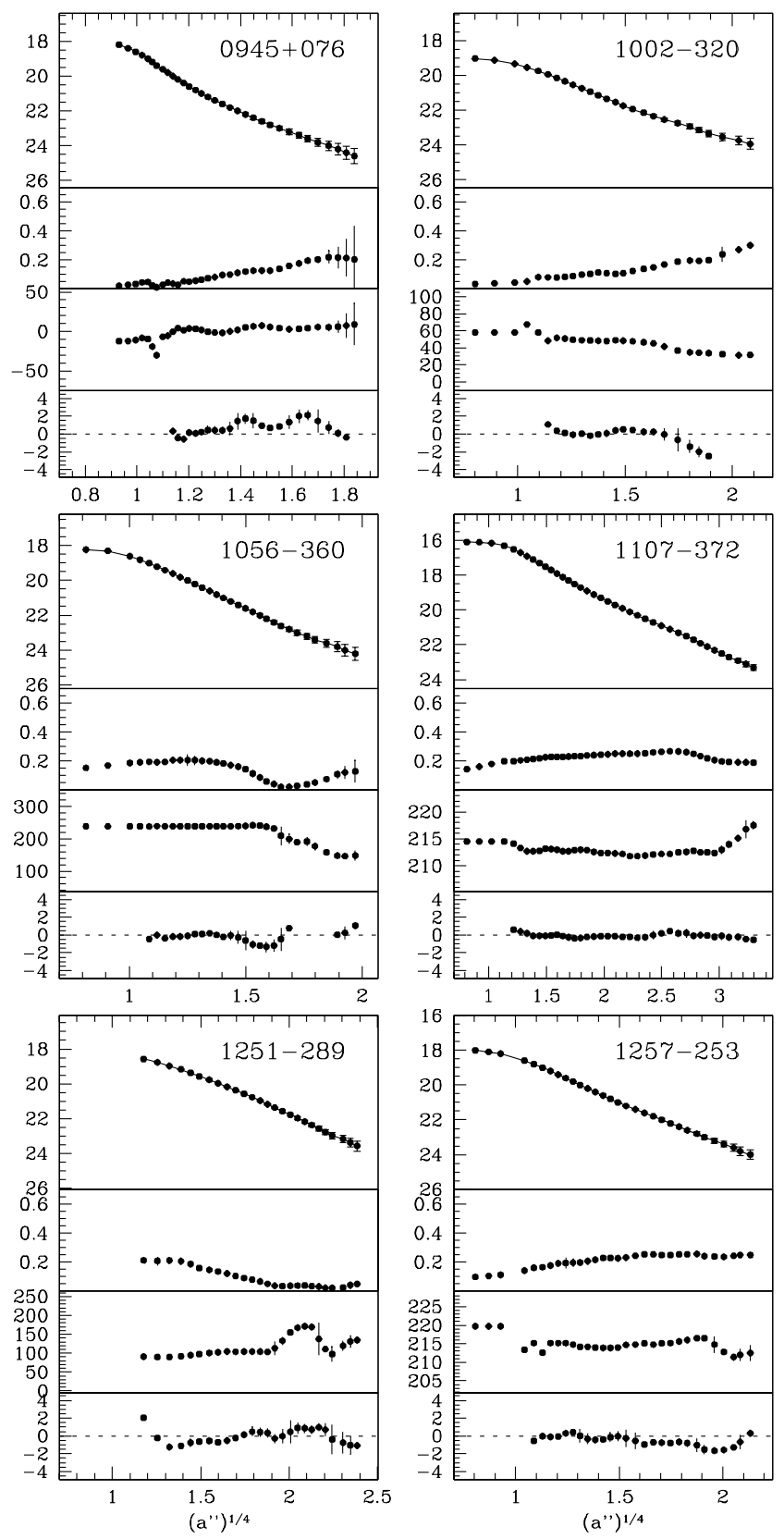

body. Both morphology and luminosity profile are consistent with the presence of a extended disk.

1056 - 360: The radio structure of this source is large and complex (Jones \& McAdam 1992). The optical counterpart lies in a rich environment and coincides with a close galaxy pair similar to $0452-190$. The radio galaxy is the brightest of the two and has normal luminosity and geometrical profiles for an elliptical galaxy. The optical spectrum exhibits emission lines (Simpson et al. 1996).

1107 - 372: NGC 3557 is an FRI radio source (Birkinshaw \& Davies 1985) in which $\mathrm{H} \alpha+[\mathrm{NII}]$ extended 

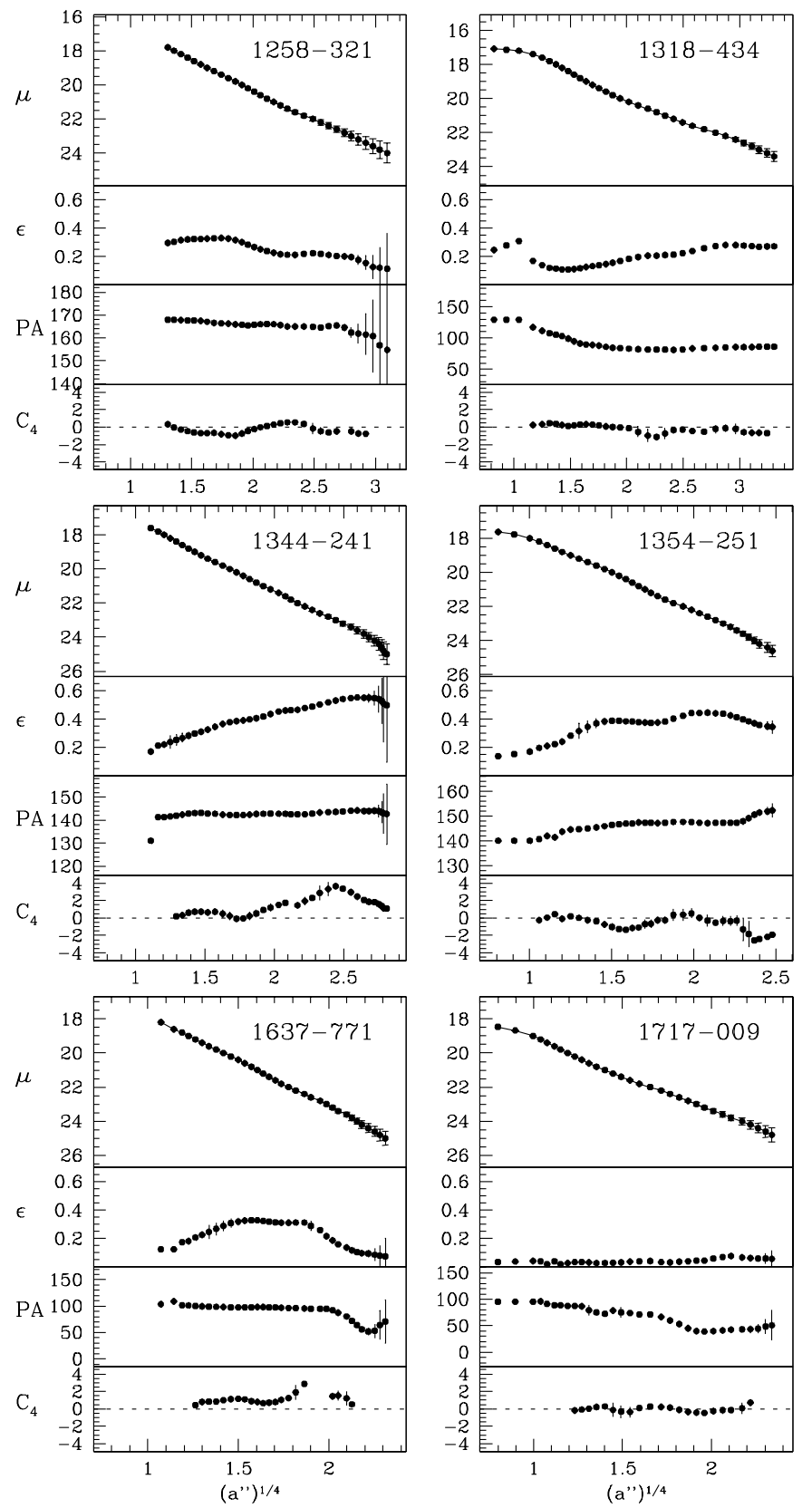

Fig. 3. continued

emission has been detected (Goudfrooij et al. 1994). The luminosity and geometrical profiles indicate a regularelliptical morphology.

1123 - 351: Its optical counterpart is a large elliptical galaxy with undisturbed morphology.

1251 - 122: 3C 278 is an FRI radio source (Morganti et al. 1993). The optical counterpart is the Southernmost component of a dumbbell system. After deblending the image of the two galaxies, we found both components are heavily disturbed by tidal interaction.
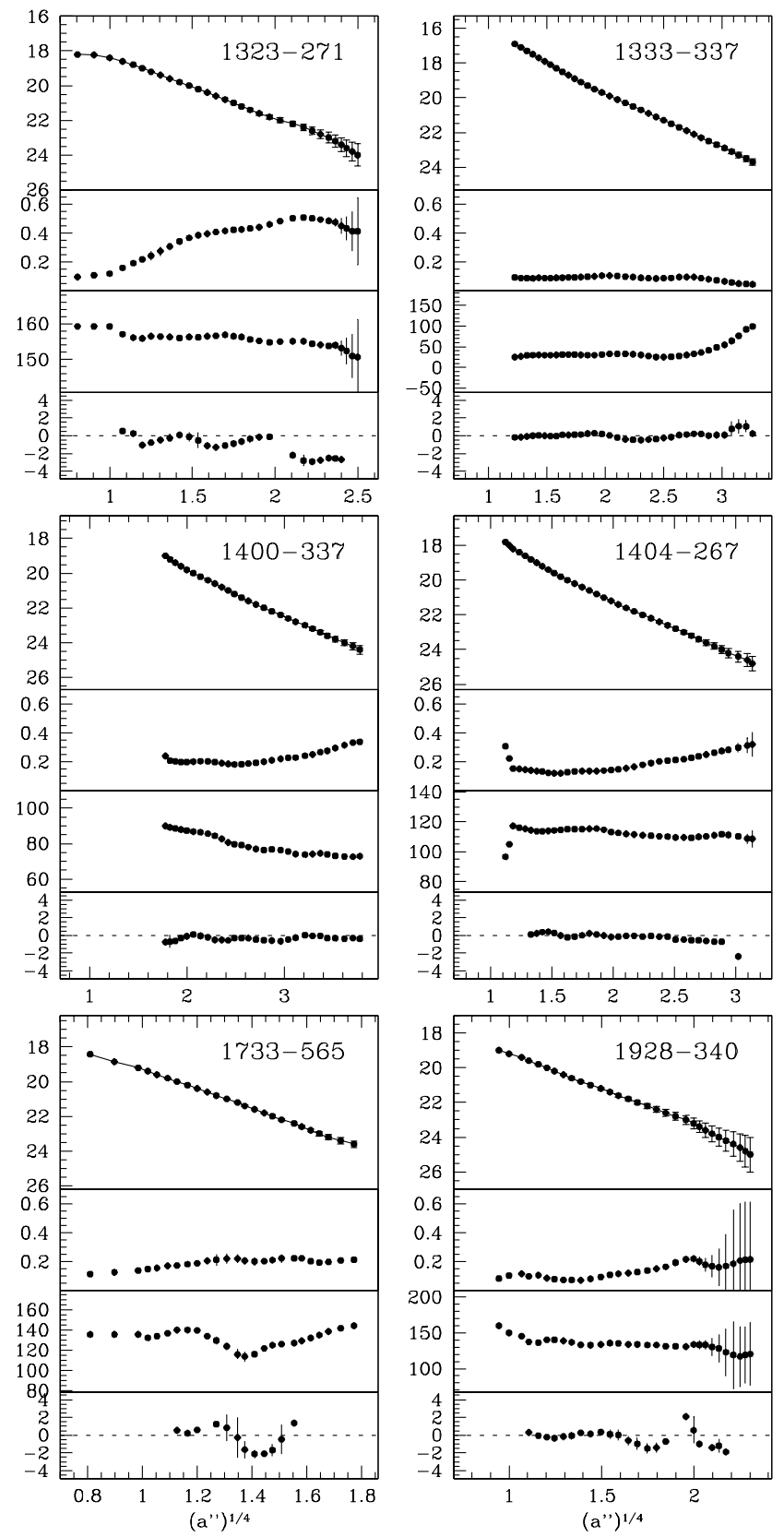

1251 - 289: The host galaxy inhabits a relatively poor environment and shows undisturbed elliptical morphology in its outer part. On the contrary, the inner region appears irregular and elongated suggesting the presence of either a nuclear dust lane or a double nucleus.

1257 - 253: An elliptical galaxy with undisturbed morphology, surrounded by several nearby companions. The optical spectrum is typical of early type galaxies without emission lines (Scarpa et al. 1996).

1258 - 321: This radio galaxy lies in the cluster Abell 3537. After masking the light from two nearby bright stars, 

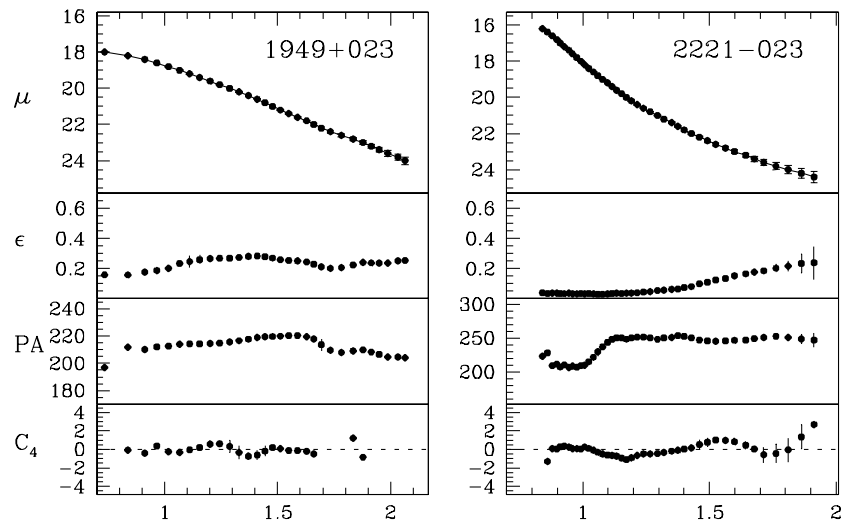

Fig. 3. continued
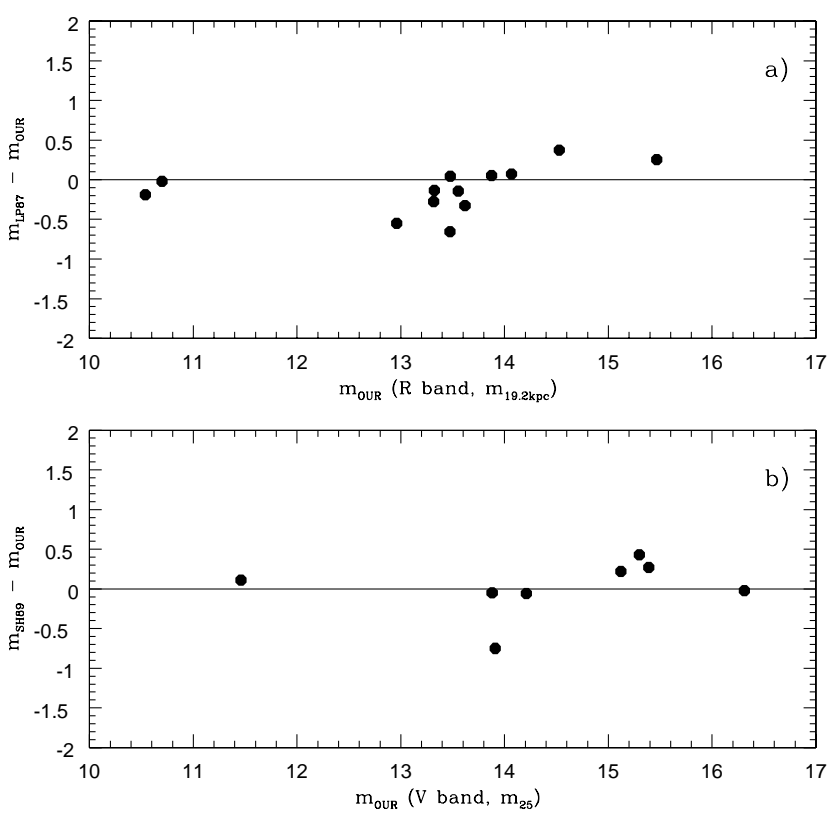

Fig. 4. a) Magnitude differences between Lilly \& Prestage (1987) and this work, versus our magnitudes. To compare these data we derived the aperture magnitudes at $19.2 \mathrm{kpc}$ from our photometry. In common with data published by LP87, the magnitudes are corrected for galactic extinction. b) Magnitude differences between Smith \& Heckman (1989a,b) and this work, versus our magnitudes. To compare these values we corrected our data for the different band of observation (assuming $V-R=0.6$ ) and transformed our total magnitudes $\left(m_{\text {tot }}\right)$ into isophotal magnitudes $\left(m_{25}\right)$. No correction for galactic extinction has been applied

the surface photometry indicates a regular elliptical morphology and a nuclear point source.

1318 - 434: NGC 5090 hosts an FRI radio source (Morganti et al. 1993) studied in detail by Lloyd et al. (1996). The galaxy looks like a normal giant elliptical, over which projects NGC 5091, an edge-on spiral.
1323 - 271: The host galaxy lies in cluster Abell 1736 and looks like a normal elliptical in interaction with a small S0 galaxy.

1333 - 337: A regular elliptical galaxy located at the center of cluster Abell 3565. Optical emission lines were detected within few arcsec of the nucleus (Goudfrooij et al. 1994).

1344 - 241: The host galaxy lies in a rather poor environment. Isophotal contours and geometrical profiles (increasing ellipticity, constant position angle and positive $c 4$ coefficient) suggest it is an S0 galaxy. However, signatures of a disc are not seen in the luminosity profile.

1354 - 251: This radio galaxy inhabits a relatively poor environment. Its optical morphology is of an undisturbed elliptical galaxy, as confirmed also by the optical spectrum (Scarpa et al. 1996).

1400 - 337: The optical counterpart is a large, regular elliptical with a bright nucleus, located at the center of the cluster Abell 753 .

1404 - 267: A large and undisturbed elliptical galaxy lying at the center of the Abell cluster 3581. Since no radio maps are available for this radio source, we guess its FRI radio morphology from the power-morphology relationship.

1637 - 771: The radio source is estimated by Morganti et al. (1993) to have an FRII radio morphology. Optical spectra have revealed the presence of relatively strong emission lines (Tadhunter et al. 1993; Simpson et al. 1996). The luminosity profile of the host galaxy is consistent with the presence of a nuclear point source. The environment is rather poor.

1717 - 009: According to Morganti et al. (1993), 3C 353 is an FRII radio source. Emission lines have been detected in its optical spectrum (Tadhunter et al. 1993), and a LINER type spectrum has been also observed (Simpson et al. 1996). In our image the galaxy appears undisturbed in the outer regions, while in the center both luminosity and geometrical profiles support the existence of a bright point source.

1733 - 565: An FRII radio source (Morganti et al. 1993), with emission lines in the optical spectrum (Simpson et al. 1996). The optical counterpart is most probably an elliptical as suggested by its radial profile which precisely follows a de Vaucouleurs law. A nuclear point source is also observed.

1928 - 340: The host galaxy looks like a normal elliptical located in a small galaxy group.

1949 + 023: 3C 403 is an FRII radio source (Morganti et al. 1993) located in a poor environment. Strong emission lines were observed in its optical spectrum (Simpson et al. 1996; Tadhunter et al. 1993). In spite of the presence of a nearby bright star we derive for this galaxy a reliable profile, which clearly indicates a normal elliptical structure. 
2221 - 023: 3C 445 is a well known radio source, and broad emission lines have been observed in its optical spectrum (Eracleous \& Halpern 1994; Corbett et al. 1998). The optical morphology is characterized by the presence of an extremely bright nuclear point source.

\section{References}

Birkinshaw M., Davies R.L., 1985, ApJ 291, 32

Colina L., de Juan L., 1995, ApJ 448, 548

Corbett E.A., Robinson A., Axon D.J., Young S., Hough J.H., 1998, MNRAS 296, 721

de Juan L., Colina L., Perez-Fournon I., 1994, ApJS 91, 507

di Serego Alighieri S., Danziger I.J., Morganti R., Tadhunter C.N., 1994, MNRAS 269, 998

Ekers R.D., Wall J.V., Shaver P.A., et al., 1989, MNRAS 236, 737 (EK)

Eracleous M., Halpern J.P., 1994, ApJS 90, 1

Fanaroff B.L., Riley J.M., 1974, MNRAS 167, 31

Fasano G., 1990, Internal Report of Astr. Obs. of Padova

Fasano G., Bonoli C., 1990, A\&A 234, 89

Fasano G., Falomo R., Scarpa R., 1996, MNRAS 282, 40 (Paper I)

Gioia I.M., Luppino G.A., 1994, ApJS 94, 583

Gonzalez-Serrano J.I., Carballo R., Perez-Fournon I., 1993, AJ 105,1710

Goudfrooij P., Hansen L., Jorgensen H.E., Norgaard-Nielsen H.U., 1994, A\&AS 105, 341

Govoni F., Falomo R., Fasano G., Scarpa R., 2000, A\&A 353, 507
Hine R.G., Longair M.S., 1979, MNRAS 188, 111

Jones P.A., McAdam W.B., 1992, ApJS 80, 137

Knapp G.R., Kerr F.J., 1974, A\&A 35, 361

Landolt A.U., 1992, AJ 104, 340

Ledlow M.J., Owen F.N., 1995, AJ 109, 853

Lilly S.J., Prestage R.M., 1987, MNRAS 225, 531 (LP87)

Lloyd B.D., Jones P.A., Haynes R.F., 1996, MNRAS 279, 1197

Longair M.S., Seldner M., 1979, MNRAS 189, 433

Morganti R., Killeen N.E.B., Tadhunter C.N., 1993, MNRAS 263, 1023

Morganti R., Oosterloo T.A., Reynolds J.E., Tadhunter C.N., Migenes V., 1997, MNRAS 284, 541

Owen F.N., Laing R.A., 1989, MNRAS 238, 357

Owen F.N., White R.A., 1991, MNRAS 249, 164

Owen F.N., Odea C.P., Inoue M., Eilek J.A., 1985, ApJ 294, L85

Prestage R.M., Peacock J.A., 1988, MNRAS 230, 131

Scarpa R., Falomo R., Pesce J.E., 1996, A\&AS 116, 295

Simpson C., Ward M., Clements D.L., Rawlings S., 1996, MNRAS 281, 509

Smith E.P., Heckman T.M., 1989a, ApJS 69, 365 (SH89)

Smith E.P., Heckman T.M., 1989b, ApJ 341, 658 (SH89)

Stark A.A., Gammie C.F., Wilson R.W., et al., 1992, ApJS 79, 77

Tadhunter C.N., Morganti R., Di Serego Alighieri S., Fosbury R.A.E., Danziger I.J., 1993, MNRAS 263, 999

Wall J.V., Peacock J.A., 1985, MNRAS 216, 173 (WP)

Zensus J.A., 1989, in BL Lac objects, Maraschi L., Maccacaro T., Ulrich M.H. (eds). Berlin: Springer, p. 3 